\title{
Stability Analysis of a Vector-Borne Disease with Variable Human Population
}

\author{
Muhammad Ozair, ${ }^{1}$ Abid Ali Lashari, ${ }^{1}$ Il Hyo Jung, ${ }^{2}$ Young Il Seo, ${ }^{3}$ and Byul Nim Kim ${ }^{2}$ \\ ${ }^{1}$ Centre for Advanced Mathematics and Physics, National University of Sciences and Technology, \\ H-12, Islamabad 44000, Pakistan \\ ${ }^{2}$ Department of Mathematics, Pusan National University, Busan 609-735, Republic of Korea \\ ${ }^{3}$ National Fisheries Research and Development Institute, Busan 619-705, Republic of Korea \\ Correspondence should be addressed to Il Hyo Jung; ilhjung@pusan.ac.kr
}

Received 25 November 2012; Revised 1 March 2013; Accepted 2 March 2013

Academic Editor: Ferenc Hartung

Copyright (C) 2013 Muhammad Ozair et al. This is an open access article distributed under the Creative Commons Attribution License, which permits unrestricted use, distribution, and reproduction in any medium, provided the original work is properly cited.

\begin{abstract}
A mathematical model of a vector-borne disease involving variable human population is analyzed. The varying population size includes a term for disease-related deaths. Equilibria and stability are determined for the system of ordinary differential equations. If $R_{0} \leq 1$, the disease-"free" equilibrium is globally asymptotically stable and the disease always dies out. If $R_{0}>1$, a unique "endemic" equilibrium is globally asymptotically stable in the interior of feasible region and the disease persists at the "endemic" level. Our theoretical results are sustained by numerical simulations.
\end{abstract}

\section{Introduction}

Vector-borne diseases such as malaria, dengue fever, plague, and West Nile fever are infectious diseases caused by the influx of viruses, bacteria, protozoa, or rickettsia which are primarily transmitted by disease-transmitting biological agents, called vectors. A vector-borne disease is transmitted by a pathogenic microorganism from an infected host to another organism results to form from an infection by bloodfeeding arthropods [1].

Vector-borne diseases, in particular, mosquito-borne disease, are transmitted to humans by blood-sucker mosquitoes, which have been a big problem for the public health in the world. The literature dealing with the mathematical theory on vector-borne diseases is quite extensive. Many mathematical models concerning the emergence and reemergence of the vector-host infectious disease have been proposed and analyzed in the literature $[2,3]$.

By direct transmission models, we mean that the infection moves from person to person directly, with no environmental source, intermediate vector, or host. In a vector-host model, direct transmission may take place by transfusionrelated transmission, transplantation-related transmission, and needle-stick-related transmission [4]. Some models have been developed to study the dynamics of a vector-borne disease that considers a direct mode of transmission in human host population [5-7].

Mathematical modeling has proven to play an important role in gaining some insights into the transmission dynamics of infectious diseases and suggest control strategies. Appropriate mathematical models can provide a qualitative assessment for the problem. Some mathematical models discussed in [8-10] provide, best understanding about the dynamics and control of infectious diseases. Immense literature on the use of mathematical models for communicable diseases is available $[11,12]$. The assumption of constant population size in epidemiological models is usually valid when we study the diseases of short duration with limited effects on mortality. It may not be valid when dealing with endemic diseases such as malaria, which has a high mortality rate. Ngwa and Shu [13] assumed density-dependent death rates in both vector and human populations, so that the total populations are varying with time that includes disease-related deaths. Esteva and Vargas [14] analyzed the effect of variable host population size and disease-induced death rate. Recently Ozair et. al analyzed vector-host disease model with nonlinear incidence [15]. 
In this paper, based on the ideas posed in $[6,14]$, we develop and analyze a vector-host disease model considering a direct mode of transmission as well as a variable human population. The aim of this paper is to establish stability properties of equilibria and the threshold parameter $R_{0}$ that completely determines the existence of endemic or diseasefree equilibrium. If $R_{0} \leqslant 1$, the disease-free equilibrium is globally asymptotically stable. If $R_{0}>1$, a unique endemic equilibrium exists and is globally asymptotically stable under parametric restrictions. However, in numerical simulations it is shown that the disease still can be "endemic" even if the conditions are violated.

The rest of the paper is organized as follows. In Section 2, we present a formulation of the extended mathematical model. The dimensionless formulation of proposed model is carried out in Section 3. Section 4 devotes existence and uniqueness of "endemic" equilibria. In Section 5, we use Lyapunov function theory to show global stability of disease-"free" equilibrium and geometric approach to prove global stability of "endemic" equilibrium. Discussions and simulations are done in Section 6.

\section{Model Formulation}

The human population is partitioned into subclasses of individuals who are susceptible, infectious, and recovered, with sizes denoted by $S_{h}(t), I_{h}(t)$, and $R_{h}(t)$, respectively. The vector population is subdivided into susceptible and infectious vectors, with sizes denoted by $S_{v}(t)$ and $I_{v}(t)$, respectively. The mosquito population does not have an immune class, since their infective period ends with their death. Thus, $N_{h}(t)=S_{h}(t)+I_{h}(t)+R_{h}(t)$ and $N_{v}(t)=$ $S_{v}(t)+I_{v}(t)$ are, respectively, the total human and vector populations at time $t$. The model is given by the following system of differential equations:

$$
\begin{gathered}
\frac{d S_{h}(t)}{d t}=b_{1} N_{h}-\frac{\beta_{1} S_{h} I_{h}}{N_{h}}-\frac{\beta_{2} S_{h} I_{v}}{N_{v}}-\mu_{h} S_{h}, \\
\frac{d I_{h}(t)}{d t}=\frac{\beta_{1} S_{h} I_{h}}{N_{h}}+\frac{\beta_{2} S_{h} I_{v}}{N_{v}}-\mu_{h} I_{h}-\gamma_{h} I_{h}-\delta_{h} I_{h}, \\
\frac{d R_{h}(t)}{d t}=\gamma_{h} I_{h}-\mu_{h} R_{h}, \\
\frac{d S_{v}(t)}{d t}=\mu_{v} N_{v}-\frac{\beta_{3} S_{v} I_{h}}{N_{h}}-\mu_{v} S_{v}, \\
\frac{d I_{v}(t)}{d t}=\frac{\beta_{3} S_{v} I_{h}}{N_{h}}-\mu_{v} I_{v} .
\end{gathered}
$$

In model (1), $b_{1}$ is the recruitment rate of humans into the population which is assumed to be susceptible. Susceptible hosts get infected via two routes of transmission, through a contact with an infected individual and through being bitten by an infectious vector. We denote the infection rate of susceptible individuals which results from effective contact with infectious individuals by $\beta_{1}$ and $\beta_{2}$ is the infection rate of susceptible humans resulting due to the biting of infected vectors. The incidence of new infections via direct and indirect route of transmission is given by the standard incidence form $\beta_{1}\left(S_{h} I_{h} / N_{h}\right)$ and $\beta_{2}\left(S_{h} I_{v} / N_{h}\right)$, respectively. The term $\mu_{h}$ is the natural mortality rate of humans. We assume that infectious individuals acquire permanent immunity by the rate $\gamma_{h}$. The infectious humans suffer from diseaseinduced death at a rate $\delta_{h}$. The recruitment and natural death rate of vector population is assumed to be $\mu_{v}$. The susceptible vectors become infectious as a result of biting effect of infectious humans at a rate $\beta_{3}$, so that the incidence of newly infected vectors is again given by standard incidence form $\beta_{3}\left(S_{v} I_{h} / N_{h}\right)$. The total human population is governed by the following equation:

$$
\frac{d N_{h}}{d t}=b_{1} N_{h}-\mu_{h} N_{h}-\delta_{h} I_{h}
$$

\section{Dimensionless Formulation}

Denote $s_{h}=S_{h} / N_{h}, i_{h}=I_{h} / N_{h}, r_{h}=R_{h} / N_{h}, s_{v}=S_{v} / N_{v}$, and $i_{v}=I_{v} / N_{v}$. It is easy to verify that $s_{h}, i_{h}, r_{h}, s_{v}$, and $i_{v}$ satisfy the following system (see the Appendix details for):

$$
\begin{gathered}
\frac{d s_{h}(t)}{d t}=b_{1}\left(1-s_{h}\right)-\beta_{1} s_{h} i_{h}-\beta_{2} s_{h} i_{v}+\delta_{h} s_{h} i_{h}, \\
\frac{d i_{h}(t)}{d t}=\beta_{1} s_{h} i_{h}+\beta_{2} s_{h} i_{v}-\left(b_{1}+\gamma_{h}+\delta_{h}\right) i_{h}+\delta_{h} i_{h}^{2}, \\
\frac{d r_{h}(t)}{d t}=\gamma_{h} i_{h}-b_{1} r_{h}+\delta_{h} i_{h} r_{h}, \\
\frac{d s_{v}(t)}{d t}=\mu_{v}\left(1-s_{v}\right)-\beta_{3} s_{v} i_{h}, \\
\frac{d i_{v}(t)}{d t}=\beta_{3} s_{v} i_{h}-\mu_{v} i_{v},
\end{gathered}
$$

where solutions are restricted to $s_{h}+i_{h}+r_{h}=1$ and $s_{v}+i_{v}=1$. Before analyzing the unnormalized model (1) and (2), we consider the normalized model (3) by scaling, and so we can study the following reduced system that describes the dynamics of the proportion of individuals in each class

$$
\begin{gathered}
\frac{d s_{h}(t)}{d t}=b_{1}\left(1-s_{h}\right)-\beta_{1} s_{h} i_{h}-\beta_{2} s_{h} i_{v}+\delta_{h} s_{h} i_{h}, \\
\frac{d i_{h}(t)}{d t}=\beta_{1} s_{h} i_{h}+\beta_{2} s_{h} i_{v}-\left(b_{1}+\gamma_{h}+\delta_{h}\right) i_{h}+\delta_{h} i_{h}^{2}, \\
\frac{d i_{v}(t)}{d t}=\beta_{3}\left(1-i_{v}\right) i_{h}-\mu_{v} i_{v},
\end{gathered}
$$

determining $r_{h}$ from

$$
\frac{d r_{h}(t)}{d t}=\gamma_{h} i_{h}-b_{1} r_{h}+\delta_{h} i_{h} r_{h}
$$

or from $r_{h}=1-s_{h}-i_{h}$ and $s_{v}$ from $s_{v}=1-i_{v}$, respectively. The correlation between normalized and unnormalized models is explained in the Appendix. Throughout this work, we study the reduced system (4) in the closed, positively invariant set $\Gamma=\left\{\left(s_{h}, i_{h}, i_{v}\right) \in R_{+}^{3}, 0 \leq s_{h}+i_{h} \leq 1,0 \leq i_{v} \leq 1\right\}$, where $R_{+}^{3}$ denotes the nonnegative cone of $R^{3}$ with its lower dimensional faces. 


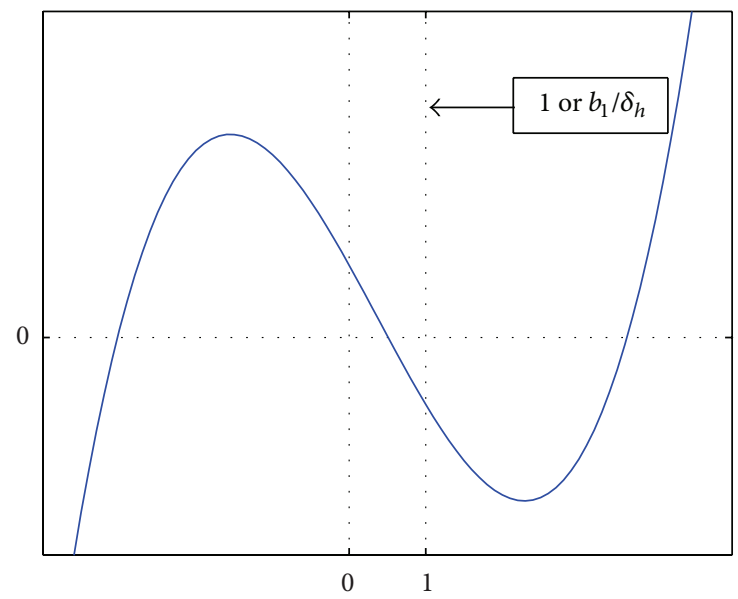

FIGURE 1: $\beta_{1}>\delta_{h}$.

\section{Existence of Equilibria}

We seek the conditions for the existence and stability of the disease-"free" equilibrium (DFE) $E_{0}\left(s_{h 0}, 0,0\right)$ and the "endemic" proportion equilibrium $E^{*}\left(s_{h}^{*}, i_{h}^{*}, i_{v}^{*}\right)$. Obviously, $E_{0}(1,0,0) \in \Gamma$ is the DFE of (4), which exists for all positive parameters. The Jacobian matrix of (4) at an arbitrary point $E\left(s_{h}, i_{h}, i_{v}\right)$ takes the following form:

$J(E)$

$=\left(\begin{array}{ccc}-b_{1}-\beta_{1} i_{h}-\beta_{2} i_{v}+\delta_{h} i_{h} & -\left(\beta_{1}-\delta_{h}\right) s_{h} & -\beta_{2} s_{h} \\ \beta_{1} i_{h}+\beta_{2} i_{v} & \beta_{1} s_{h}-\left(b_{1}+\gamma_{h}+\delta_{h}\right)+2 \delta_{h} i_{h} & \beta_{2} s_{h} \\ 0 & \beta_{3}\left(1-i_{v}\right) & -\beta_{3} i_{h}-\mu_{v}\end{array}\right)$.

To analyze the stability of DFE, we calculate the characteristic equation of $J(E)$ at $E=E_{0}$ as follows:

$$
\begin{aligned}
\left(\lambda+b_{1}\right)\left(\lambda^{2}+\right. & \lambda\left(\mu_{v}+b_{1}+\gamma_{h}+\delta_{h}-\beta_{1}\right) \\
& \left.+\mu_{v}\left(b_{1}+\gamma_{h}+\delta_{h}\right)\left(1-R_{0}\right)\right),
\end{aligned}
$$

where

$$
R_{0}=\frac{\beta_{1}}{b_{1}+\gamma_{h}+\delta_{h}}+\frac{\beta_{2} \beta_{3}}{\mu_{v}\left(b_{1}+\gamma_{h}+\delta_{h}\right)} .
$$

By Routh Hurwitz criteria [16], all roots of (7) have negative real parts if and only if $R_{0}<1$. So, $E_{0}$ is locally asymptotically stable for $R_{0}<1$. If $R_{0}>1$, the characteristic equation (7) has positive eigenvalue, and $E_{0}$ is thus unstable. We established the following theorem.

Theorem 1. The disease-free equilibrium is locally asymptotically stable whenever $R_{0}<1$ and unstable for $R_{0}>1$.
In order to find the "endemic" equilibrium of (4), we set the right hand side of (4) equal to zero and get

$$
\begin{gathered}
i_{v}^{*}=\frac{\beta_{3} i_{h}^{*}}{\mu_{v}+\beta_{3} i_{h}^{*}}, \\
s_{h}^{*}=\frac{b_{1}\left(\mu_{v}+\beta_{3} i_{h}^{*}\right)}{\left(\mu_{v}+\beta_{3} i_{h}^{*}\right)\left(b_{1}+\left(\beta_{1}-\delta_{h}\right) i_{h}^{*}\right)+\beta_{2} \beta_{3} i_{h}^{*}},
\end{gathered}
$$

where $i_{h}^{*}$ is a positive solution of the equation

$$
f\left(i_{h}^{*}\right)=A_{3} i_{h}^{* 3}+A_{2} i_{h}^{* 2}+A_{1} i_{h}^{*}+A_{0}=0,
$$

where

$$
\begin{gathered}
A_{3}=\beta_{3} \delta_{h}\left(\beta_{1}-\delta_{h}\right), \\
A_{2}=\beta_{2} \beta_{3} \delta_{h}+\left(\mu_{v} \delta_{h}-\beta_{3}\left(b_{1}+\gamma_{h}+\delta_{h}\right)\right)\left(\beta_{1}-\delta_{h}\right) \\
+b_{1} \beta_{3} \delta_{h}, \\
A_{1}=b_{1}\left(\mu_{v} \delta_{h}+\beta_{1} \beta_{3}\right) \\
-\left(b_{1}+\gamma_{h}+\delta_{h}\right)\left(\beta_{2} \beta_{3}+b_{1} \beta_{3}+\mu_{v}\left(\beta_{1}-\delta_{h}\right)\right), \\
A_{0}=\left(b_{1}+\gamma_{h}+\delta_{h}\right) b_{1} \mu_{v}\left(R_{0}-1\right) .
\end{gathered}
$$

From right hand side of (5), we have $\gamma_{h} i_{h}^{*}=\left(b_{1}-\delta_{h} i_{h}^{*}\right) r_{h}^{*}>0$ and second equation of (9) $\beta_{1} \mu_{v}+\beta_{2} \beta_{3}-\mu_{v} \delta_{h}>\beta_{3} \delta_{h} i_{h}^{*}$, which means that

$$
0<i_{h}^{*}<\min \left\{1, \frac{b_{1}}{\delta_{h}},\left(\frac{\beta_{1} \mu_{v}+\beta_{2} \beta_{3}}{\mu_{v} \delta_{h}}-1\right) \frac{\mu_{v}}{\beta_{3}}\right\} .
$$

If $\left(\beta_{1} \mu_{v}+\beta_{2} \beta_{3}\right) / \mu_{v} \delta_{h} \leq 1$, there is no positive $i_{h}^{*}$, and therefore the only equilibrium point in $\Gamma$ is $E_{0}$. Note that this is a special case of $R_{0}<1$.

Assume that $R_{0}>1$.

(1) If $\beta_{1}>\delta_{h}$, then $A_{3}>0$, we have $f(-\infty)<0, f(\infty)>$ 0 and $f(0)=A_{0}>0$. Further, $f(1)<0$ (if $b_{1} / \delta_{h} \geq 1$ ) and $f\left(b_{1} / \delta_{h}\right)<0$. Thus, there exists unique $i_{h}^{*}$ such that $f\left(i_{h}^{*}\right)=0$ (see Figure 1$)$.

(2) If $\beta_{1}=\delta_{h}$, then $A_{3}=0$ and $f\left(i_{h}^{*}\right)=A_{2} i_{h}^{* 2}+A_{1} i_{h}^{*}+$ $A_{0}$, where $A_{2}=\beta_{2} \beta_{3} \delta_{h}+b_{1} \beta_{3} \delta_{h}>0$. We observe that $f(-\infty)>0, f(\infty)>0$ and $f(0)=A_{0}>0$. Moreover, $f(1)<0$ (if $b_{1} / \delta_{h} \geq 1$ ) and $f\left(b_{1} / \delta_{h}\right)<0$. Therefore, there exists unique $i_{h}^{*}$ such that $f\left(i_{h}^{*}\right)=0$ (see Figure 2).

(3) If $\beta_{1}<\delta_{h}$, then $A_{3}<0$, we have $f(-\infty)>0, f(\infty)<$ 0 and still $f(0)=A_{0}>0, f(1)<0$ (if $b_{1} / \delta_{h} \geq 1$ ), $f\left(b_{1} / \delta_{h}\right)<0$. In this case, we can say that there is only one root or three roots in the interval $(0,1)$ if $b_{1} / \delta_{h} \geq$ 1 or $\left(0, b_{1} / \delta_{h}\right)$ if $b_{1} / \delta_{h}<1$.

We know that $f\left(i_{h}^{*}\right)=0$ has three real roots if and only if

$$
\frac{q^{2}}{4}+\frac{p^{3}}{27} \leq 0
$$




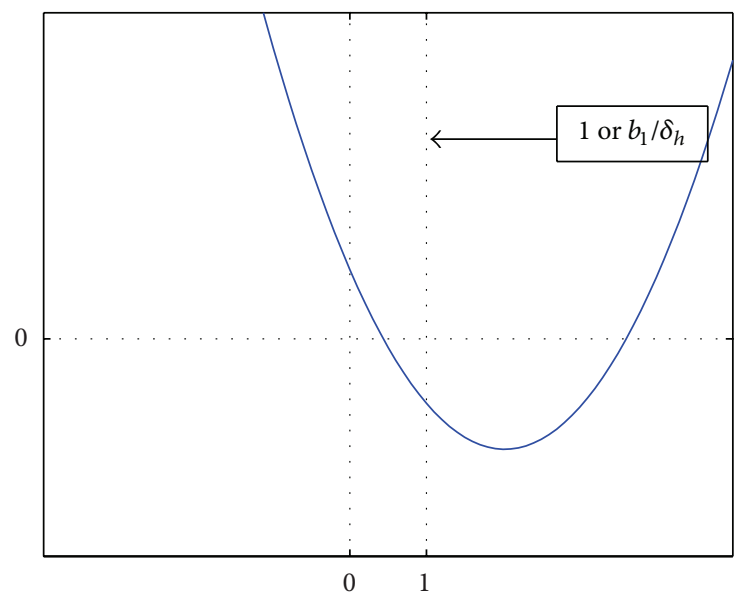

Figure 2: $\beta_{1}=\delta_{h}$.

where

$$
\begin{gathered}
p=\frac{A_{1}}{A_{3}}-\frac{\left(A_{2}\right)^{2}}{3\left(A_{3}\right)^{2}}, \\
q=\frac{A_{0}}{A_{3}}-\frac{A_{1} A_{2}}{3\left(A_{3}\right)^{2}}+\frac{2\left(A_{2}\right)^{3}}{27\left(A_{3}\right)^{3}},
\end{gathered}
$$

or

$$
\widehat{R}_{1}=\frac{18 A_{0} A_{1} A_{2} A_{3}-4 A_{0}\left(A_{2}\right)^{3}-4\left(A_{1}\right)^{3} A_{3}+\left(A_{1}\right)^{2}\left(A_{2}\right)^{2}}{27\left(A_{0}\right)^{2}\left(A_{3}\right)^{2}}
$$$$
\geq 1 \text {. }
$$

If $\widehat{R}_{1}<1$, there is unique $i_{h}^{*}$ such that $f\left(i_{h}^{*}\right)=0$ in the feasible interval.

If $\widehat{R}_{1}>1$, there are three different real roots for $f\left(i_{h}^{*}\right)=0$ say $i_{h 1}^{*}, i_{h 2}^{*}, i_{h 3}^{*}\left(i_{h 1}^{*}<i_{h 2}^{*}<i_{h 3}^{*}\right)$. Note that, differentiating with respect to $i_{h}^{*}$, we obtain

$$
f^{\prime}\left(i_{h}^{*}\right)=3 A_{3} i_{h}^{* 2}+2 A_{2} i_{h}^{*}+A_{1} .
$$

The three different real roots for $f\left(i_{h}^{*}\right)=0$ are in the feasible interval if and only if the following inequalities are satisfied:

$$
\begin{gathered}
0<\frac{-A_{2}}{3 A_{3}}<1, \\
f^{\prime}(0)=A_{1}<0, \\
f^{\prime}(1)=3 A_{3}+2 A_{2}+A_{1}<0\left(\text { if } \frac{b_{1}}{\delta_{h}} \geq 1\right), \\
f^{\prime}\left(\frac{b_{1}}{\delta_{h}}\right)=3 A_{3}\left(\frac{b_{1}}{\delta_{h}}\right)^{2}+2 A_{2}\left(\frac{b_{1}}{\delta_{h}}\right)+A_{1}<0\left(\text { if } \frac{b_{1}}{\delta_{h}}<1\right) .
\end{gathered}
$$

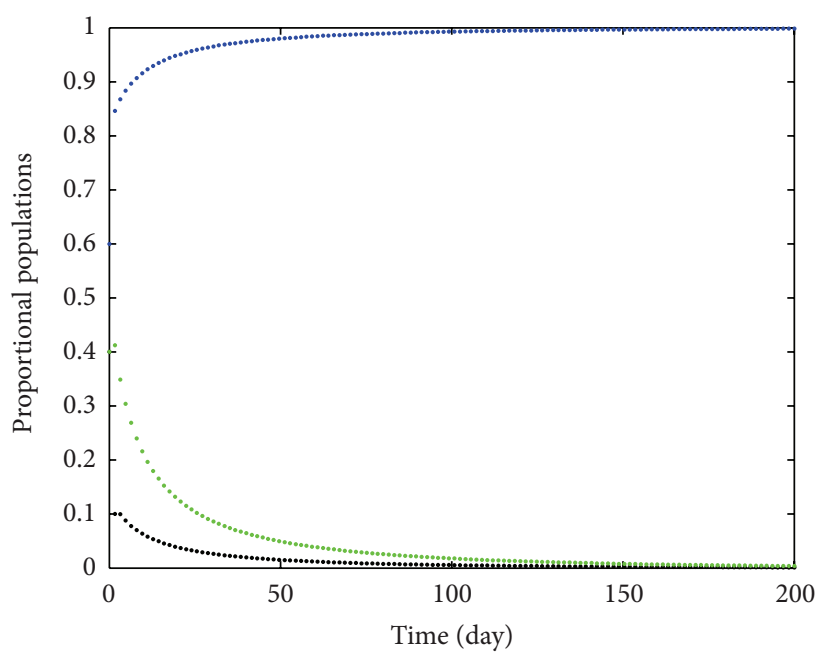

$$
\begin{aligned}
& -s_{h} \\
& \text { - } i_{h} \\
& \text { - } i_{v}
\end{aligned}
$$

FIGURE $3: b_{1}=1 ; \beta_{1}=0.02 ; \beta_{2}=0.4 ; \beta_{3}=0.6 ; \gamma_{h}=0.3 ; \mu_{v}=0.2$; $\beta_{1}=\delta_{h}=0.02 ; R_{0}=0.92$.

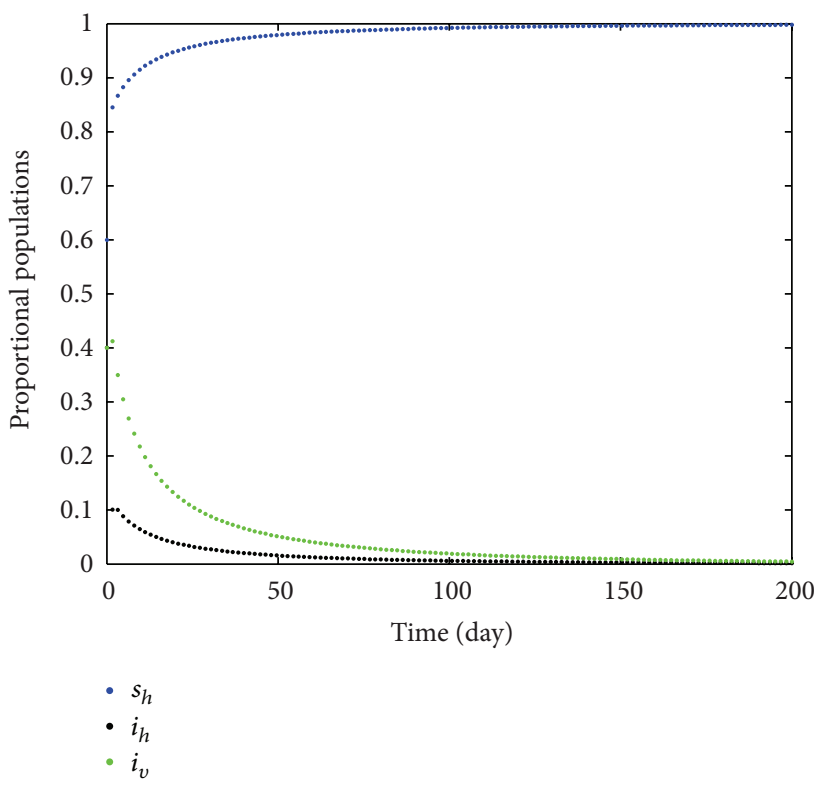

FIGURE $4: b_{1}=1 ; \beta_{1}=0.02 ; \beta_{2}=0.4 ; \beta_{3}=0.6 ; \gamma_{h}=0.3 ; \mu_{v}=0.2$; $\beta_{1}>\delta_{h}=0.01 ; R_{0}=0.93$.

If $\widehat{R}_{1}=1$, there are three real roots for $f\left(i_{h}^{*}\right)=0$, in which at least two are identical. Similarly, if inequalities (17) are satisfied, then there are three real roots for $f\left(i_{h}^{*}\right)=0$ in the feasible interval, say $i_{h 1}^{*}, i_{h 2}^{*}, i_{h 3}^{*}\left(i_{h 1}^{*}=i_{h 2}^{*}\right)$.

Assume that $R_{0}=1$.

(1) If $\beta_{1}=\delta_{h}$, then $A_{3}=0$ and (10) reduces to $i_{h}^{*}\left(A_{2} i_{h}^{*}+A_{1}\right)=0$, which implies that $i_{h}^{*}=0$ or $i_{h}^{*}=-A_{1} / A_{2}$, which is positive but it lies outside the interval $(0,1)$ if $b_{1} / \delta_{h} \geq 1$ or $\left(0, b_{1} / \delta_{h}\right)$ if $b_{1} / \delta_{h}<1$. 


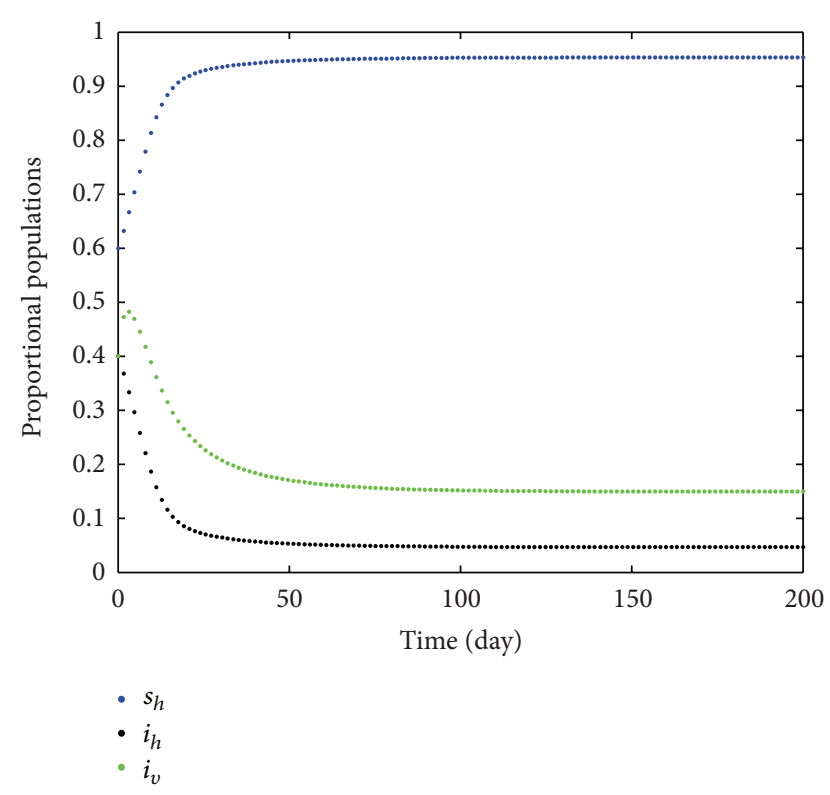

FIGURE $5: b_{1}=2 ; \beta_{1}=0.025 ; \beta_{2}=0.65 ; \beta_{3}=0.75 ; \gamma_{h}=0.000045$; $\mu_{v}=0.2 ; \beta_{1}>\delta_{h}=0.000025 ; R_{0}=1.23$.

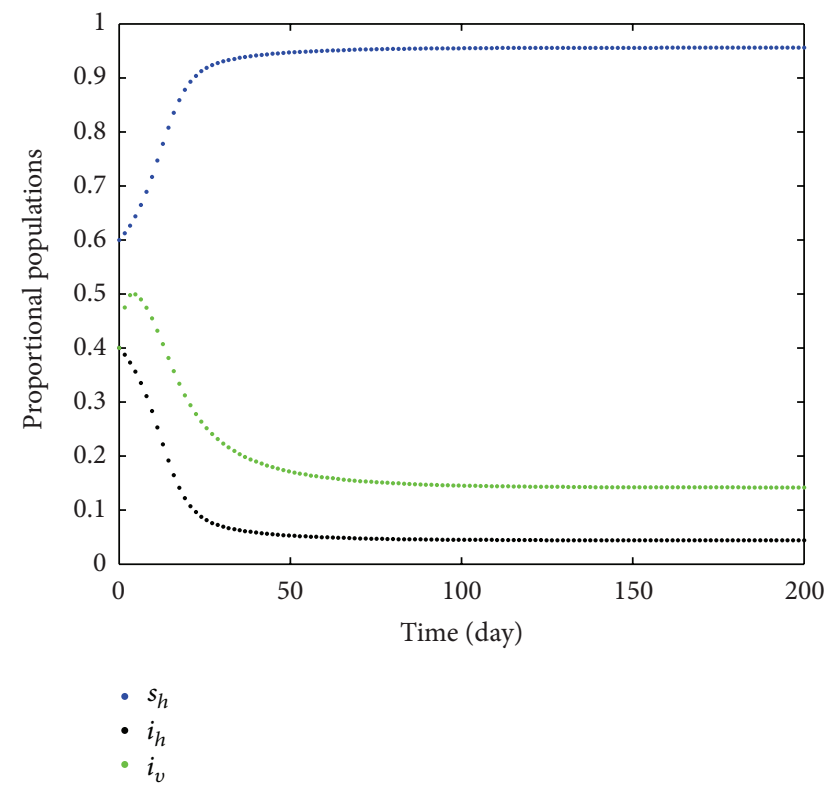

FIGURE $6: b_{1}=2 ; \beta_{1}=0.0025 ; \beta_{2}=0.65 ; \beta_{3}=0.75 ; \gamma_{h}=0.000045$; $\mu_{v}=0.2 ; \beta_{1}=\delta_{h}=0.0025 ; R_{0}=1.22$.

(2) If $\beta_{1}>\delta_{h}$, then $A_{3}>0$, we have $i_{h}^{*}\left(A_{3} i_{h}^{* 2}+A_{2} i_{h}^{*}+\right.$ $\left.A_{1}\right)=0$, which implies that $i_{h}^{*}=0$ or $i_{h}^{*}$ is the solution of the equation

$$
g\left(i_{h}^{*}\right)=A_{3} i_{h}^{* 2}+A_{2} i_{h}^{*}+A_{1}=0
$$

where $g(-\infty)>0, g(\infty)>0$, and $g(0)=A_{1}<0$. Moreover, $g(1)<0$ (if $\left.b_{1} / \delta_{h} \geq 1\right)$ and $g\left(b_{1} / \delta_{h}\right)<$ 0 if $b_{1} / \delta_{h}<1$. Therefore, there exists no $i_{h}^{*}$ such that $g\left(i_{h}^{*}\right)=0$ in the interval $(0,1)$ if $b_{1} / \delta_{h} \geq 1$ or $\left(0, b_{1} / \delta_{h}\right)$ if $b_{1} / \delta_{h}<1$.
In summary, regarding the existence and the number of the "endemic" equilibria, we have the following.

Theorem 2. Suppose that $\beta_{1} \geq \delta_{h}$. There is always a disease"free" equilibrium for system (4); if $R_{0}>1$, then there is a unique "endemic" equilibrium $E^{*}\left(s_{h}^{*}, i_{h}^{*}, i_{v}^{*}\right)$ with coordinates satisfying (9) and (10) besides the disease-"free" equilibrium.

\section{Global Dynamics}

5.1. Global Stability of the Disease- "Free" Equilibrium. In this subsection, we analyze the global behavior of the equilibria for system (4). The following theorem provides the global property of the disease-free equilibrium $E_{0}$ of the system.

Theorem 3. If $R_{0} \leq 1$, then the infection-free equilibrium $E_{0}$ is globally asymptotically stable in the interior of $\Gamma$.

Proof. To establish the global stability of the disease-free equilibrium, we construct the following Lyapunov function:

$$
L(t)=\mu_{v} i_{h}(t)+\beta_{2} i_{v}(t) .
$$

Calculating the time derivative of $L$ along (4), we obtain

$$
\begin{aligned}
L^{\prime}(t)= & \mu_{v} i_{h}^{\prime}(t)+\beta_{2} i_{v}^{\prime}(t) \\
= & \mu_{v}\left[\beta_{1} s_{h} i_{h}+\beta_{2} s_{h} i_{v}-\left(b_{1}+\gamma_{h}+\delta_{h}\right) i_{h}\right. \\
& \left.+\delta_{h} i_{h}^{2}\right]+\beta_{2}\left(\beta_{3} s_{v} i_{h}-\mu_{v} i_{v}\right) \\
= & \mu_{v}\left[\beta_{1}\left(1-i_{h}\right) i_{h}+\beta_{2}\left(1-i_{h}\right) i_{v}-\left(b_{1}+\gamma_{h}+\delta_{h}\right) i_{h}\right. \\
& \left.\quad+\delta_{h} i_{h}^{2}\right]+\beta_{2}\left[\beta_{3}\left(1-i_{v}\right) i_{h}-\mu_{v} i_{v}\right] \\
= & \mu_{v}\left[\beta_{1} i_{h}-\beta_{1} i_{h}^{2}+\beta_{2} i_{v}-\beta_{2} i_{h} i_{v}-\left(b_{1}+\gamma_{h}+\delta_{h}\right) i_{h}\right. \\
& \left.\quad+\delta_{h} i_{h}^{2}\right]+\beta_{2}\left(\beta_{3} i_{h}-\beta_{3} i_{v} i_{h}-\mu_{v} i_{v}\right) \\
= & \mu_{v} \beta_{1} i_{h}-\mu_{v} \beta_{1} i_{h}^{2}+\mu_{v} \beta_{2} i_{v}-\mu_{v} \beta_{2} i_{h} i_{v} \\
& -\mu_{v}\left(b_{1}+\gamma_{h}+\delta_{h}\right) i_{h}+\mu_{v} \delta_{h} i_{h}^{2}+\beta_{2} \beta_{3} i_{h}-\beta_{2} \beta_{3} i_{v} i_{h} \\
& -\beta_{2} \mu_{v} i_{v} \\
= & -\mu_{v}\left(b_{1}+\gamma_{h}+\delta_{h}\right)\left(1-R_{0}\right) i_{h}-\mu_{v}\left(\beta_{1}-\delta_{h}\right) i_{h}^{2} \\
& -\mu_{v} \beta_{2} i_{h} i_{v}-\beta_{2} \beta_{3} i_{v} i_{h} .
\end{aligned}
$$

Thus, $L^{\prime}(t)$ is negative if $R_{0} \leq 1$ and $L^{\prime}=0$ if and only if $i_{h}=0$. Consequently, the largest compact invariant set in $\left\{\left(S_{h}, I_{h}, I_{v}\right) \in \Gamma, L^{\prime}=0\right\}$, when $R_{0} \leq 1$, is the singelton $\left\{E_{0}\right\}$. Hence, LaSalle's invariance principle [16] implies that " $E_{0}$ " is globally asymptotically stable in $\Gamma$. This completes the proof.

5.2. Global Stability of "Endemic" Equilibrium. Here, we use the geometrical approach of $\mathrm{Li}$ and Muldowney to investigate the global stability of the endemic equilibrium $E^{*}$ in the 


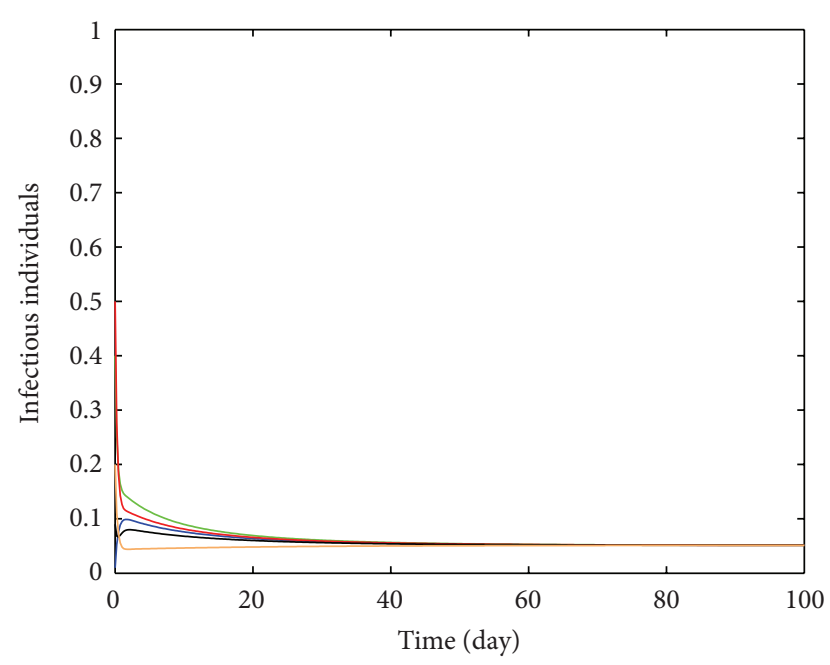

FigURE $7: b_{1}=2 ; \beta_{1}=0.4 ; \beta_{2}=0.85 ; \beta_{3}=0.75 ; \gamma_{h}=0.85 ; \mu_{v}=0.2$; $\delta_{h}=0.0000001<\beta_{1}<b_{1} / 2=1, \gamma_{h} / 2=0.425 ; R_{0}=1.26$.

feasible region $\Gamma$. We have omitted the detailed introduction of this approach, and we refer the interested readers to see [17]. We summarize this approach below.

Consider a $C^{1}$ map $f: x \mapsto f(x)$ from an open set $D \subset$ $R^{n}$ to $R^{n}$ such that each solution $x\left(t, x_{0}\right)$ to the differential equation

$$
x^{\prime}=f(x) .
$$

is uniquely determined by the initial value $x\left(0, x_{0}\right)$. We have the following assumptions:

$\left(H_{1}\right) D$ is simply connected;

$\left(\mathrm{H}_{2}\right)$ there exists a compact absorbing set $K \subset D$;

$\left(H_{3}\right)(21)$ has unique equilibrium $\bar{x}$ in $D$.

Let $P: x \mapsto P(x)$ be a nonsingular $\left(\begin{array}{c}n \\ 2\end{array}\right) \times\left(\begin{array}{c}n \\ 2\end{array}\right)$ matrixvalued function which is $C^{1}$ in $D$ and a vector norm $|\cdot|$ on $R^{N}$, where $N=\left(\begin{array}{l}n \\ 2\end{array}\right)$.

Let $\mu$ be the Lozinskii measure with respect to the $|\cdot|$. Define a quantity $\bar{q}_{2}$ as

$$
\bar{q}_{2}=\limsup _{t \rightarrow \infty} \sup _{x_{0} \in K} \frac{1}{t} \int_{0}^{t} \mu\left(B\left(x\left(s, x_{0}\right)\right)\right) d s,
$$

where $B=P_{f} P^{-1}+P J^{[2]} P^{-1}$, the matrix $P_{f}$ is obtained by replacing each entry $p$ of $P$ by its derivative in the direction of $f,\left(p_{i j}\right)_{f}$, and $J^{[2]}$ is the second additive compound matrix of the Jacobian matrix $J$ of (21). The following result has been established in Li and Muldowney [17].

Theorem 4. Suppose that $\left(H_{1}\right),\left(H_{2}\right)$, and $\left(H_{3}\right)$ hold, the unique endemic equilibrium $E^{*}$ is globally stable in $\Gamma$ if $\bar{q}_{2}<0$.

Obviously $\Gamma$ is simply connected and $E^{*}$ is a unique endemic equilibrium for $R_{0}>1$ in $\Gamma$. To apply the result of

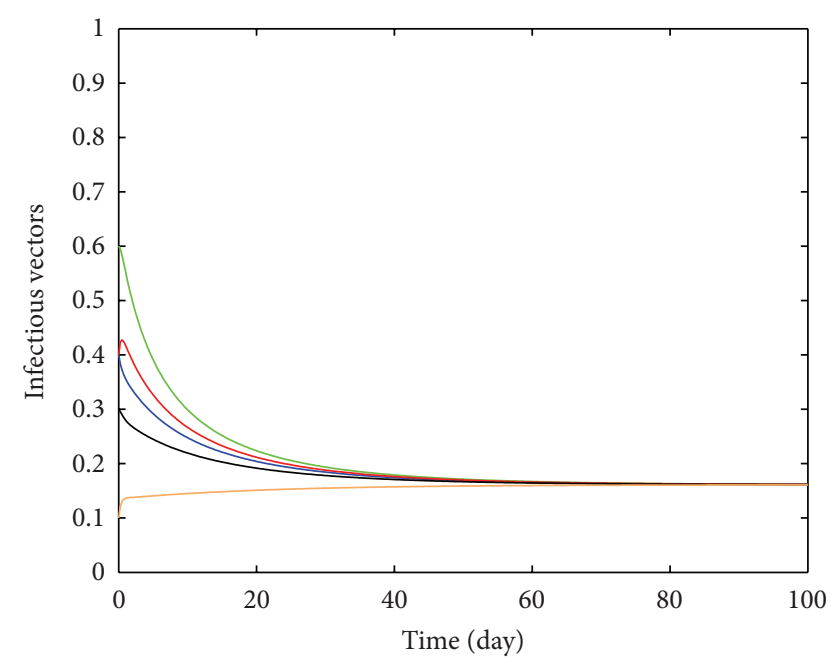

FIGURE $8: b_{1}=2 ; \beta_{1}=0.4 ; \beta_{2}=0.85 ; \beta_{3}=0.75 ; \gamma_{h}=0.85 ; \mu_{v}=0.2$; $\delta_{h}=0.0000001<\beta_{1}<b_{1} / 2=1, \gamma_{h} / 2=0.425 ; R_{0}=1.26$.

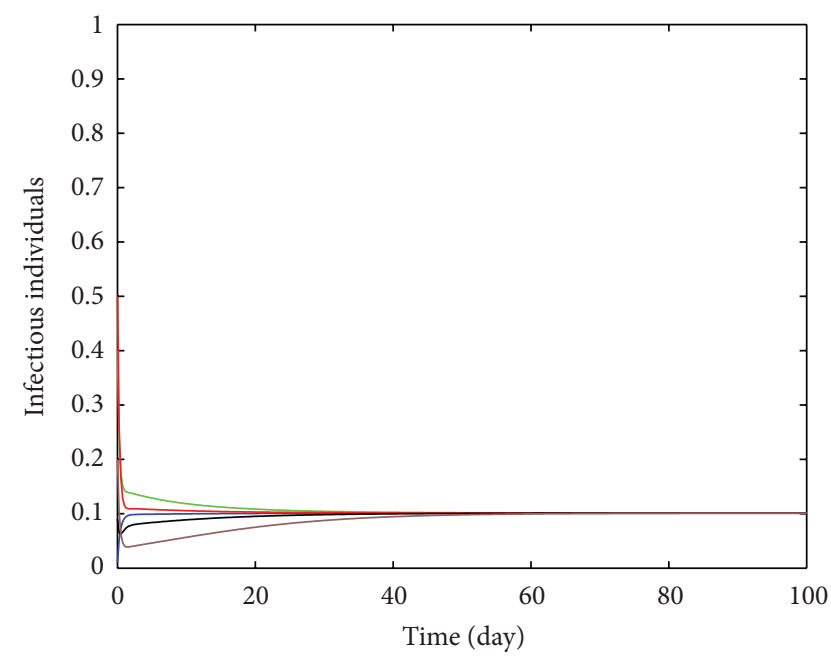

FIGURE 9: $b_{1}=2 ; \beta_{1}=0.04 ; \beta_{2}=0.85 ; \beta_{3}=0.65 ; \gamma_{h}=0.85 ; \mu_{v}=0.1$; $0.04=\delta_{h}=\beta_{1}<b_{1} / 2=1, \gamma_{h} / 2=0.425 ; R_{0}=1.92$.

the above theorem for global stability of endemic equilibrium $E^{*}$, we first prove the uniform persistence of (4) when the threshold parameter $R_{0}>1$, by applying the acyclicity Theorem (see [18]).

Definition 5 (see [19]). The system (4) is uniformly persistent, that is, there exists $c>0$ (independent of initial conditions), such that $\liminf _{t \rightarrow \infty} s_{h} \geq c, \liminf _{t \rightarrow \infty} i_{h} \geq$ $c, \liminf _{t \rightarrow \infty} i_{v} \geq c$.

Let $X$ be a locally compact metric space with metric $d$ and let $\Gamma$ be a closed nonempty subset of $X$ with boundary $\Gamma$ and interior $\Gamma^{\circ}$. Clearly, $\Gamma^{\circ}$ is a closed subset of $\Gamma$. Let $\Phi_{t}$ be a dynamical system defined on $\Gamma$. A set $B$ in $X$ is said to be invariant if $\Phi(B, t)=B$. Define $M_{\partial}:=\{x \in \Gamma: \Phi(t, x) \in$ $\Gamma$, for all $t \geq 0$ \}. 


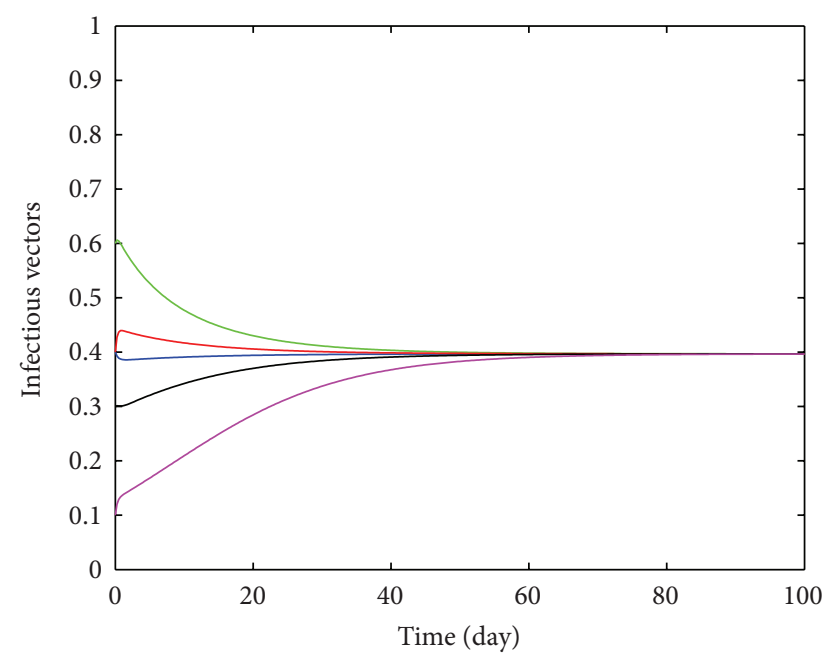

FigURE 10: $b_{1}=2 ; \beta_{1}=0.04 ; \beta_{2}=0.85 ; \beta_{3}=0.65 ; \gamma_{h}=0.85$; $\mu_{v}=0.1 ; 0.04=\delta_{h}=\beta_{1}<b_{1} / 2=1, \gamma_{h} / 2=0.425 ; R_{0}=1.92$.

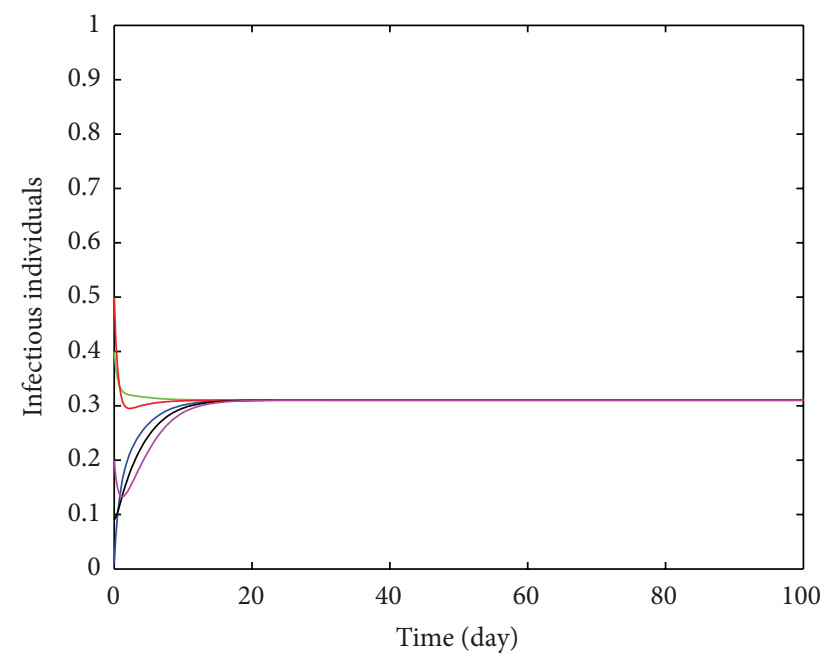

FIGURE $11: b_{1}=1 ; \beta_{1}=0.01 ; \beta_{2}=0.85 ; \beta_{3}=0.95 ; \gamma_{h}=0.015$; $\mu_{v}=0.25 ; 0.009=\delta_{h}, \gamma_{h} / 2=0.0075<\beta_{1}<b_{1} / 2=0.5 ; R_{0}=3.16$.

\section{Lemma 6 (see [18]). Assume that}

(a) $\Phi_{t}$ has a global attractor;

(b) there exists $M=\left\{M_{1}, \ldots, M_{k}\right\}$ of pair-wise disjoint, compact and isolated invariant set on $\partial \Gamma$ such that

(1) $\bigcup_{x \in M_{\partial}} \omega(x) \subseteq \bigcup_{j=1}^{k} M_{j}$;

(2) no subsets of $M$ form a cycle on $\partial \Gamma$;

(3) each $M_{j}$ is also isolated in $\Gamma$;

(4) $W^{s}\left(M_{j}\right) \cap \Gamma^{\circ}=\phi$ for each $1 \leq j \leq k$, where $W^{s}\left(M_{j}\right)$ is stable manifold of $M_{j}$. Then $\Phi_{t}$ is uniformly persistent with respect to $\Gamma$.

Proof. We have $\Gamma=\left\{\left(s_{h}, i_{h}, i_{v}\right) \in R_{+}^{3}, 0 \leq s_{h}+i_{h} \leq 1,0 \leq i_{v} \leq\right.$ $1\}, \Gamma^{\circ}=\left\{\left(s_{h}, i_{h}, i_{v}\right) \in R_{+}^{3} s_{h}, i_{h}>0\right\}, \partial \Gamma=\Gamma / \Gamma^{\circ}$. Obviously

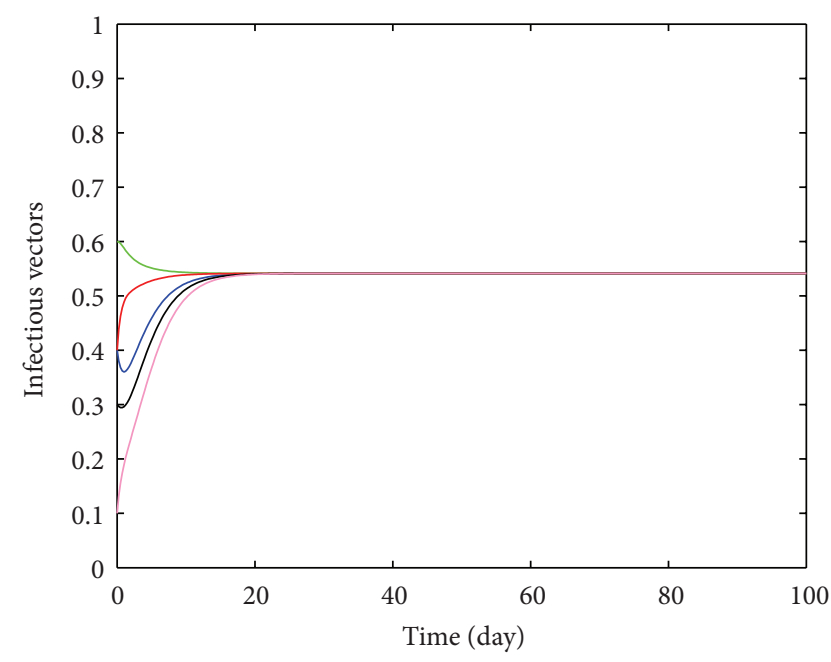

FIGURE $12: b_{1}=1 ; \beta_{1}=0.01 ; \beta_{2}=0.85 ; \beta_{3}=0.95 ; \gamma_{h}=0.015$; $\mu_{v}=0.25 ; 0.009=\delta_{h}, \gamma_{h} / 2=0.0075<\beta_{1}<b_{1} / 2=0.5 ; R_{0}=3.16$.

$M_{\partial}=\partial \Gamma$. Since $\Gamma$ is bounded and positively invariant, so there exists a compact set $M$ in which all solutions of system (4) initiated in $\Gamma$ ultimately enter and remain forever. On $s_{h}$-axis we have $s_{h}^{\prime}=b_{1}\left(1-s_{h}\right)$ which means $s_{h} \rightarrow 1$ as $t \rightarrow \infty$. Thus, $E_{0}$ is the only omega limit point on $\partial \Gamma$, that is, $\omega(x)=E_{0}$ for all $x \in M_{\partial}$. Furthermore, $M=E_{0}$ is a covering of $\Omega=\bigcup_{x \in M_{\partial}} \omega(x)$, because all solutions initiated on the $s_{h}$-axis converge to $E_{0}$. Also $E_{0}$ is isolated and acyclic. This verifies that hypothesis (1) and (2) hold. When $R_{0}>1$, the disease-"free" equilibrium (DFE) $E_{0}$ is unstable from Theorem 1 and also $W^{s}(M)=\partial Г$. Hypothesis (3) and (4) hold. Therefore, there always exists a global attractor due to ultimate boundedness of solutions.

The boundedness of $\Gamma$ and the above lemma imply that (4) has a compact absorbing set $K \subset \Gamma$ [19]. Now we shall prove that the quantity $\bar{q}_{2}<0$. We choose a suitable vector norm $|\cdot|$ in $R^{3}$ and a $3 \times 3$ matrix-valued function

$$
P(x)=\left(\begin{array}{ccc}
1 & 0 & 0 \\
0 & \frac{i_{h}}{i_{v}} & 0 \\
0 & 0 & \frac{i_{h}}{i_{v}}
\end{array}\right) .
$$

Obviously, $P$ is $C^{1}$ and nonsingular in the interior of $\Omega$. Linearizing system (4) about an endemic equilibrium $E^{*}$ gives the following Jacobian matrix:

$$
\begin{aligned}
& J\left(E^{*}\right) \\
& =\left(\begin{array}{ccc}
-\frac{b_{1}}{s_{h}} & -\left(\beta_{1}-\delta_{h}\right) s_{h} & -\beta_{2} s_{h} \\
\beta_{1} i_{h}+\beta_{2} i_{v} & \beta_{1} s_{h}-\left(b_{1}+\gamma_{h}+\delta_{h}\right)+2 \delta_{h} i_{h} & \beta_{2} s_{h} \\
0 & \beta_{3}\left(1-i_{v}\right) & -\beta_{3} i_{h}-\mu_{v}
\end{array}\right) .
\end{aligned}
$$




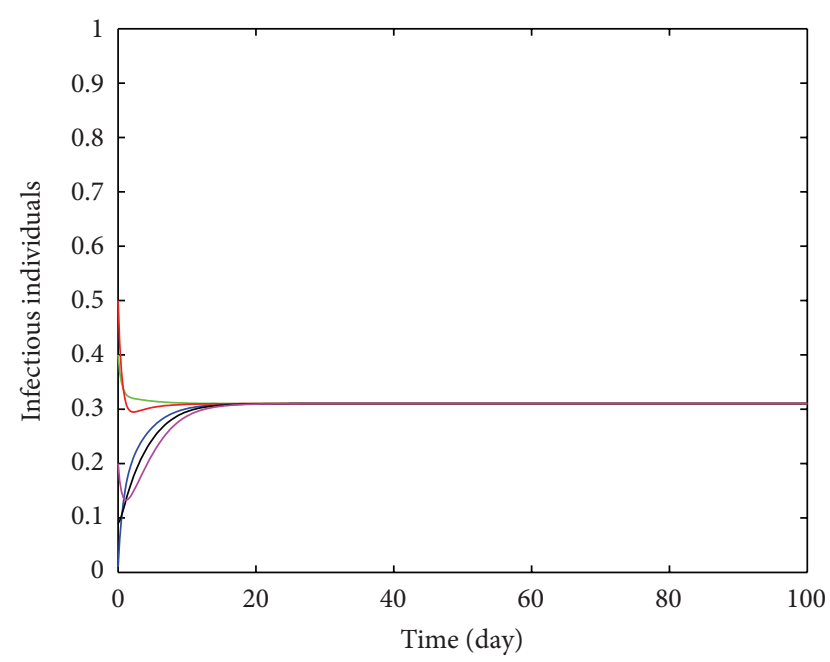

FIGURE 13: $b_{1}=1 ; \beta_{1}=0.01 ; \beta_{2}=0.85 ; \beta_{3}=0.95 ; \gamma_{h}=0.015$; $\mu_{v}=0.25 ; \gamma_{h} / 2=0.0075<0.01=\delta_{h}=\beta_{1}<b_{1} / 2=0.5 ; R_{0}=3.16$.

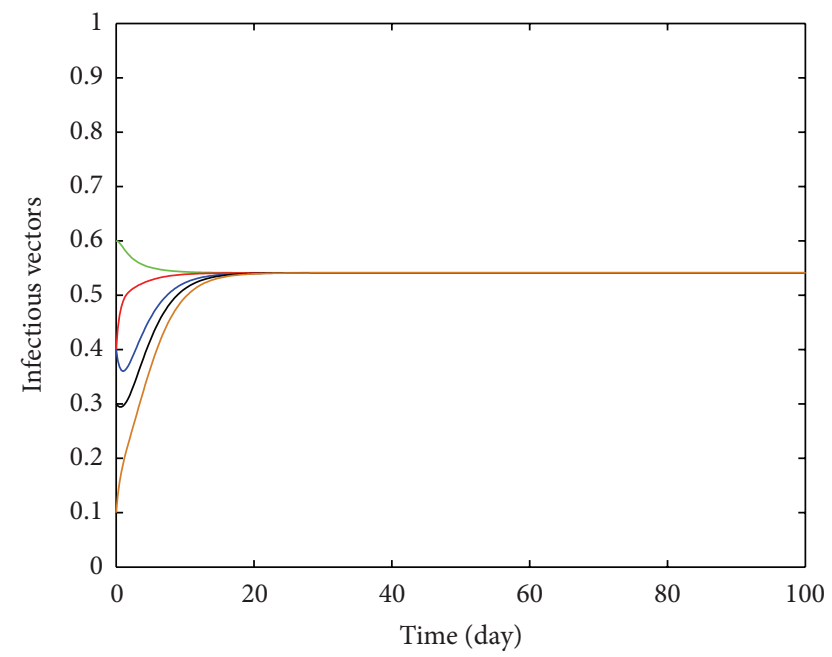

FIGURE $14: b_{1}=1 ; \beta_{1}=0.01 ; \beta_{2}=0.85 ; \beta_{3}=0.95 ; \gamma_{h}=0.015$; $\mu_{v}=0.25 ; \gamma_{h} / 2=0.0075<0.01=\delta_{h}=\beta_{1}<b_{1} / 2=0.5 ; R_{0}=3.16$.

The second additive compound matrix of $J\left(E^{*}\right)$ is given by

$$
J^{[2]}=\left(\begin{array}{ccc}
M_{11} & \beta_{2} s_{h} & \beta_{2} s_{h} \\
\beta_{3}\left(1-i_{v}\right) & M_{22} & -\left(\beta_{1}-\delta_{h}\right) s_{h} \\
0 & \beta_{1} i_{h}+\beta_{2} i_{v} & M_{33}
\end{array}\right),
$$

where

$$
\begin{gathered}
M_{11}=-\frac{b_{1}}{s_{h}}+\beta_{1} s_{h}-\left(b_{1}+\gamma_{h}+\delta_{h}\right)+2 \delta_{h} i_{h}, \\
M_{22}=-\frac{b_{1}}{s_{h}}-\beta_{3} i_{h}-\mu_{v}, \\
M_{33}=\beta_{1} s_{h}-\left(b_{1}+\gamma_{h}+\delta_{h}\right)+2 \delta_{h} i_{h}-\beta_{3} i_{h}-\mu_{v} .
\end{gathered}
$$

The matrix $B=P_{f} P^{-1}+P J^{[2]} P^{-1}$ can be written in block form as

$$
B=\left(\begin{array}{ll}
B_{11} & B_{12} \\
B_{21} & B_{22}
\end{array}\right)
$$

with

$$
\begin{gathered}
B_{11}=-\frac{b_{1}}{s_{h}}+\beta_{1} s_{h}-\left(b_{1}+\gamma_{h}+\delta_{h}\right)+2 \delta_{h} i_{h}, \\
B_{12}=\left(\beta_{2} s_{h} \frac{i_{v}}{i_{h}}, \beta_{2} s_{h} \frac{i_{v}}{i_{h}}\right), \\
B_{21}=\left(\begin{array}{c}
\left(\frac{i_{h}}{i_{v}}\right) \beta_{3}\left(1-i_{v}\right) \\
0
\end{array}\right), \\
B_{22}=\left(\begin{array}{ll}
Q_{11} & Q_{12} \\
Q_{21} & Q_{22}
\end{array}\right),
\end{gathered}
$$

where

$$
\begin{gathered}
Q_{11}=-\frac{b_{1}}{s_{h}}-\beta_{3} i_{h}-\mu_{v}, \\
Q_{12}=-\left(\beta_{1}-\delta_{h}\right) s_{h}, \\
Q_{21}=\beta_{1} i_{h}+\beta_{2} i_{v}, \\
Q_{22}=\beta_{1} s_{h}-\left(b_{1}+\gamma_{h}+\delta_{h}\right)+2 \delta_{h} i_{h}-\beta_{3} i_{h}-\mu_{v}, \\
\frac{i_{v}}{i_{h}}\left(\frac{i_{h}}{i_{v}}\right)_{f}=\frac{i_{h}^{\prime}}{i_{h}}-\frac{i_{v}^{\prime}}{i_{v}} .
\end{gathered}
$$

Consider the norm in $R^{3}$ as $|(u, v, w)|=\max (|u|,|v|+$ $|w|)$ where $(u, v, w)$ denotes the vector in $R^{3}$. The Lozinskiur, measure with respect to this norm is defined as $\mu(B) \leq$ $\sup \left(g_{1}, g_{2}\right)$, where

$$
g_{1}=\mu_{1}\left(B_{11}\right)+\left|B_{12}\right|, \quad g_{2}=\mu_{1}\left(B_{22}\right)+\left|B_{21}\right| .
$$

From system (4), we can write

$$
\begin{gathered}
\frac{i_{h}^{\prime}}{i_{h}}=\beta_{1} s_{h}+\beta_{2} s_{h} \frac{i_{v}}{i_{h}}-\left(b_{1}+\gamma_{h}+\delta_{h}\right)+\delta_{h} i_{h}, \\
\frac{i_{v}^{\prime}}{i_{v}}=\beta_{3}\left(1-i_{v}\right) \frac{i_{h}}{i_{v}}-\mu_{v} .
\end{gathered}
$$

Since $B_{11}$ is a scalar, its Lozinskii measure with respect to any vector norm in $R^{1}$ will be equal to $B_{11}$. Thus

$$
\begin{gathered}
B_{11}=-\frac{b_{1}}{s_{h}}+\beta_{1} s_{h}-\left(b_{1}+\gamma_{h}+\delta_{h}\right)+2 \delta_{h} i_{h}, \\
\left|B_{12}\right|=\beta_{2} s_{h} \frac{i_{v}}{i_{h}},
\end{gathered}
$$

and $g_{1}$ will become

$$
\begin{aligned}
g_{1} & =-\frac{b_{1}}{s_{h}}+\beta_{1} s_{h}-\left(b_{1}+\gamma_{h}+\delta_{h}\right)+2 \delta_{h} i_{h}+\beta_{2} s_{h} \frac{i_{v}}{i_{h}} \\
& =\frac{i_{h}^{\prime}}{i_{h}}-\frac{b_{1}}{s_{h}}+\delta_{h} i_{h} \\
& \leq \frac{i_{h}^{\prime}}{i_{h}}-b_{1}+\delta_{h} i_{h} .
\end{aligned}
$$


Also $\left|B_{21}\right|=\left(i_{h} / i_{v}\right) \beta_{3}\left(1-i_{v}\right),\left|B_{12}\right|$ and $\left|B_{21}\right|$ are the operator norms of $B_{12}$ and $B_{21}$ which are mapping from $R^{2}$, to $R$ and from $R$ to $R^{2}$ respectively, and $R^{2}$ is endowed with the $l_{1}$ norm. $\mu_{1}\left(B_{22}\right)$ is the Lozinskir measure of $2 \times 2$ matrix $B_{22}$ with respect to $l_{1}$ norm in $R^{2}$;

$$
\begin{aligned}
\mu\left(B_{22}\right)=\operatorname{Sup}\left\{\frac{i_{v}}{i_{h}}\left(\frac{i_{h}}{i_{v}}\right)_{f}-\frac{b_{1}}{s_{h}}-\beta_{3} i_{h}-\mu_{v}+\beta_{1} i_{h}\right. \\
+\beta_{2} i_{v}, \frac{i_{v}}{i_{h}}\left(\frac{i_{h}}{i_{v}}\right)_{f}+\left(\beta_{1}-\delta_{h}\right) s_{h}+\beta_{1} s_{h} \\
\left.\quad-\left(b_{1}+\gamma_{h}+\delta_{h}\right)+2 \delta_{h}-\beta_{3} i_{h}-\mu_{v} i_{h}\right\} \\
\leq \frac{i_{h}^{\prime}}{i_{h}}-\frac{i_{v}^{\prime}}{i_{v}}-b_{1}+\delta_{h} i_{h}-\beta_{3} i_{h}-\mu_{v},
\end{aligned}
$$

if $\beta_{1} \leq \gamma_{h} / 2$. Hence

$$
\begin{aligned}
g_{2} & \leq \frac{i_{h}^{\prime}}{i_{h}}-\frac{i_{v}^{\prime}}{i_{v}}-b_{1}+\delta_{h} i_{h}-\beta_{3} i_{h}-\mu_{v}+\left(\frac{i_{h}}{i_{v}}\right) \beta_{3}\left(1-i_{v}\right) \\
& =\frac{i_{h}^{\prime}}{i_{h}}-b_{1}+\delta_{h} i_{h}-\beta_{3} i_{h} .
\end{aligned}
$$

Thus,

$$
\begin{aligned}
\mu(B) & =\operatorname{Sup}\left\{g_{1}, g_{2}\right\} \\
& \leq \frac{i_{h}^{\prime}}{i_{h}}-b_{1}+\delta_{h} \\
& \leq \frac{i_{h}^{\prime}}{i_{h}}-\bar{\beta}_{1},
\end{aligned}
$$

where $\bar{\beta}_{1}=\min \left(\gamma_{h} / 2, b_{1} / 2\right)$. Since (4) is uniformly persistent when $R_{0}>1$, so for $T>0$ such that $t>T$ implies $i_{h}(t) \geq c, i_{v}(t) \geq c$ and $(1 / t) \log i_{h}(t)<\left(\bar{\beta}_{1} / 2\right)$ for all $\left(s_{h}(0), i_{h}(0), i_{v}(0)\right) \in K$. Thus,

$$
\frac{1}{t} \int_{0}^{t} \mu(B) d t<\frac{\log i_{h}(t)}{t}-\bar{\beta}_{1}<-\frac{\bar{\beta}_{1}}{2},
$$

for all $\left(s_{h}(0), i_{h}(0), i_{v}(0)\right) \in K$, which further implies that $\bar{q}_{2}<$ 0 . Therefore, all the conditions of Theorem 4 are satisfied. Hence, unique endemic equilibrium $E^{*}$ is globally stable in $\Gamma$.

\section{Discussions and Simulations}

This paper deals with a vector-host disease model which allows a direct mode of transmission and varying human population. It concerns diseases with long duration and substantial mortality rate (e.g., malaria). Our main results are concerned with the global dynamics of transformed proportionate system. We have constructed Lyapunov function

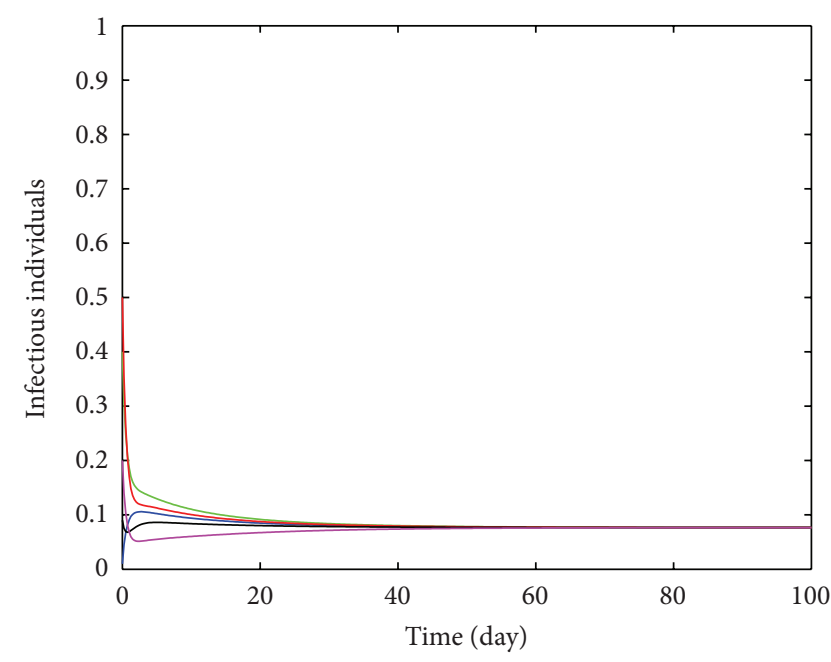

FIGURE 15: $b_{1}=0.78 ; \beta_{1}=0.4 ; \beta_{2}=0.65 ; \beta_{3}=0.55 ; \gamma_{h}=0.8$; $\mu_{v}=0.15 ; 0.35=\delta_{h}, b_{1} / 2=0.39<\beta_{1}<\gamma_{h} / 2=0.0075 ; R_{0}=1.44$.

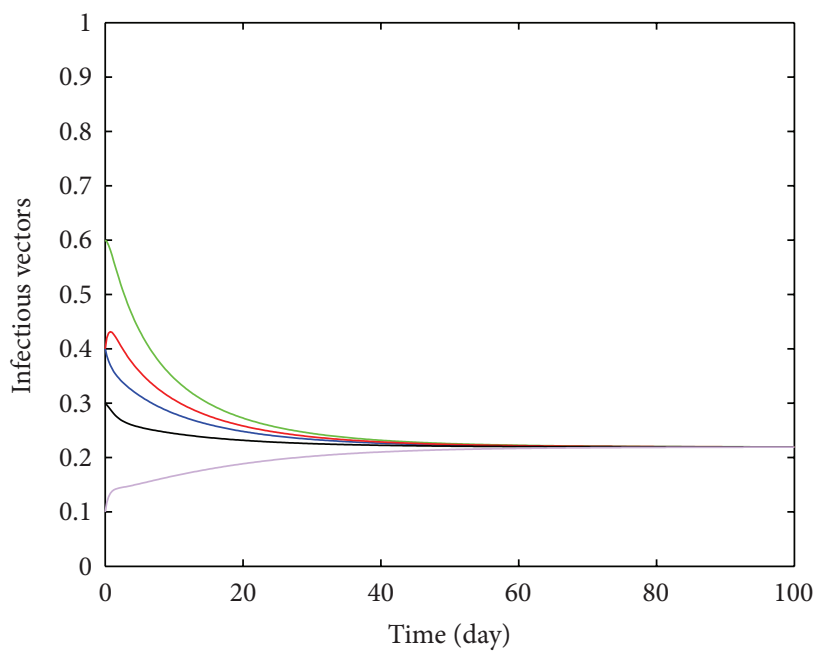

FIGURE $16: b_{1}=0.78 ; \beta_{1}=0.4 ; \beta_{2}=0.65 ; \beta_{3}=0.55 ; \gamma_{h}=0.8$; $\mu_{v}=0.15 ; 0.35=\delta_{h}, b_{1} / 2=0.39<\beta_{1}<\gamma_{h} / 2=0.0075 ; R_{0}=1.44$.

to show the global stability of disease-"free" equilibrium and the geometric approach is used to prove the global stability of "endemic" equilibrium. The epidemiological correlations between the two systems (normalized and unnormalized) have also been discussed. The dynamical behavior of the proportionate model is determined by the basic reproduction number of the disease. The model has a globally asymptotically stable disease-"free" equilibrium whenever $R_{0} \leq 1$ (Figures 3 and 4). When $R_{0}>1$, the disease persists at an "endemic" level (Figures 5 and 6) if $\beta_{1}<\min \left(b_{1} / 2, \gamma_{h} / 2\right)$. Figures 7, 8, 9, and 10 describe numerically "endemic" level of infectious individuals and infectious vectors under the condition $\beta_{1}<\min \left(b_{1} / 2, \gamma_{h} / 2\right)$. We here question that what are the dynamics of the proportionate system (4) even if the condition $\beta_{1}<\min \left(b_{1} / 2, \gamma_{h} / 2\right)$ is not satisfied? We see numerically that if $\delta_{h}, \gamma_{h} / 2<\beta_{1}<b_{1} / 2$ or $\gamma_{h} / 2<\beta_{1}=\delta_{h}<$ $b_{1} / 2$ then infectious individuals and infectious vectors will 


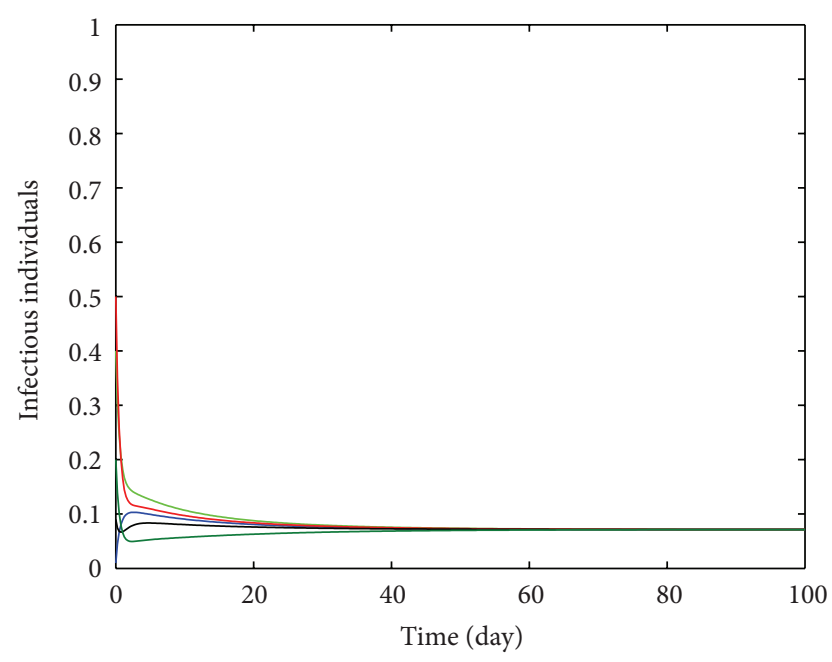

FIGURE 17: $b_{1}=0.78 ; \beta_{1}=0.4 ; \beta_{2}=0.65 ; \beta_{3}=0.55 ; \gamma_{h}=0.8$; $\mu_{v}=0.15 ; b_{1} / 2=0.39<0.4=\delta_{h}=\beta_{1}<\gamma_{h} / 2=0.0075 ; R_{0}=1.40$.

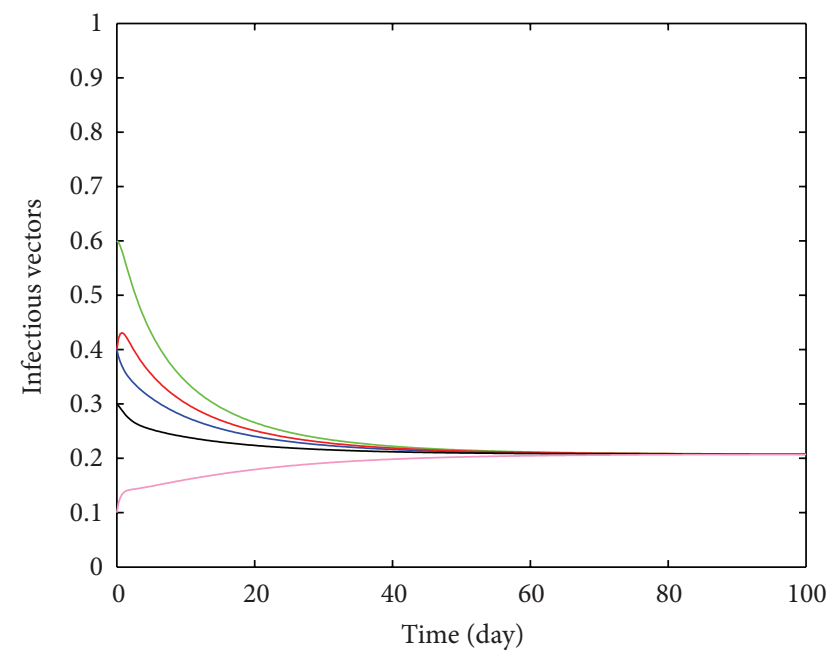

FIGURE 18: $b_{1}=0.78 ; \beta_{1}=0.4 ; \beta_{2}=0.65 ; \beta_{3}=0.55 ; \gamma_{h}=0.8$; $\mu_{v}=0.15 ; b_{1} / 2=0.39<0.4=\delta_{h}=\beta_{1}<\gamma_{h} / 2=0.0075 ; R_{0}=1.40$.

also approach to endemic level for different initial conditions (Figures 11,12,13, and 14). It is also numerically shown that the same is true for the case $\delta_{h}, b_{1} / 2<\beta_{1}<\gamma_{h} / 2$ or $b_{1} / 2<\beta_{1}=$ $\delta_{h}<\gamma_{h} / 2$ (Figures 15, 16, 17, and 18). This implies that the condition $\beta_{1}<\min \left(b_{1} / 2, \gamma_{h} / 2\right)$ is weak for the global stability of unique "endemic" equilibrium.

\section{Appendix}

Using the transformation $s_{h}=S_{h} / N_{h}, i_{h}=I_{h} / N_{h}, r_{h}=$ $R_{h} / N_{h}, s_{v}=S_{v} / N_{v}$, and $i_{v}=I_{v} / N_{v}$ for scaling, their differentials: $d s_{h}(t) / d t=\left(1 / N_{h}\right)\left(d S_{h} / d t\right)-\left(S_{h} / N_{h}^{2}\right)\left(d N_{h} / d t\right)$, $d i_{h}(t) / d t=\left(1 / N_{h}\right)\left(d I_{h} / d t\right)-\left(I_{h} / N_{h}^{2}\right)\left(d N_{h} / d t\right), d r_{h}(t) / d t=$ $\left(1 / N_{h}\right)\left(d R_{h} / d t\right)-\left(R_{h} / N_{h}^{2}\right)\left(d N_{h} / d t\right), d s_{v}(t) / d t=\left(1 / N_{v}\right)$ $\left(d S_{v} / d t\right)-\left(S_{v} / N_{v}^{2}\right)\left(d N_{v} / d t\right)$, and $d i_{v}(t) / d t=\left(1 / N_{v}\right)\left(d i_{v} /\right.$ $d t)-\left(i_{v} / N_{v}^{2}\right)\left(d N_{v} / d t\right)$, and the system (1) and (2), we obtain the dimensionless form (3). If $\delta_{h}=0$ and $b_{1}=\mu_{h}$, then $N_{h}(t)^{\prime}=0$ and so $N_{h}(t)$ remains fixed at its initial value $N_{h 0}$. In this case, the system (1) becomes the model with constant population whose dynamics are the same as the proportionate system (3). Hence, the solutions with initial conditions $S_{h 0}+I_{h 0}+R_{h 0}=N_{h 0}$ tend to $\left(N_{h 0}, 0,0\right)$ if $R_{0} \leq 1$ and to $N_{h 0}\left(s_{h}^{*}, i_{h}^{*}, r_{h}^{*}\right)$ if $R_{0}>1$. In the rest of this section, we suppose that $\delta_{h}>0$. From system (1) and (2), the trivial equilibrium $E=(0,0,0,0,0)$ can be easily obtained. Assume that $E_{*}=\left(N_{h}^{*}, S_{h}^{*}, I_{h}^{*}, R_{h}^{*}, I_{v}^{*}\right)$ is the endemic equilibrium of system (1) and (2), where $N_{h}^{*}=S_{h}^{*}+I_{h}^{*}+R_{h}^{*}$. This equilibrium exists if and only if the following equations are satisfied

$$
\begin{gathered}
\frac{S_{h}^{*}}{N_{h}^{*}}=\frac{Q\left(\beta_{3} \alpha_{h}+\mu_{v} \delta_{h}\right)}{\beta_{1}\left(\beta_{3} \alpha_{h}+\mu_{v} \delta_{h}\right)+\beta_{2} \beta_{3} \delta_{h}}, \\
\frac{I_{h}^{*}}{N_{h}^{*}}=\frac{\alpha_{h}}{\delta_{h}}, \\
\frac{R_{h}^{*}}{N_{h}^{*}}=\frac{\gamma_{h} \alpha_{h}}{\mu_{h} \delta_{h}}, \\
\frac{I_{v}^{*}}{N_{h}^{*}}=\frac{\beta_{3} \alpha_{h} N_{v}}{\left(\beta_{3} \alpha_{h}+\mu_{v}\right) N_{h}^{*}},
\end{gathered}
$$

where $\alpha_{h}=b_{1}-\mu_{h}$ and $Q=\mu_{h}+\gamma_{h}+\delta_{h}$. We introduce the parameters

$$
\begin{gathered}
R_{1}= \begin{cases}\frac{b_{1}}{\mu_{h}}, & \text { if } R_{0} \leq 1 \\
\frac{b_{1}}{\mu_{h}+\delta_{h} i_{h}^{*}}, & \text { if } R_{0}>1,\end{cases} \\
R_{2}= \begin{cases}\frac{\beta_{1}}{\mu_{h}+\gamma_{h}+\delta_{h}}+\frac{\beta_{2} \beta_{3}}{\mu_{v}\left(\mu_{h}+\gamma_{h}+\delta_{h}\right)}, & \text { if } R_{0} \leq 1 \\
\frac{\beta_{1} s_{h}^{*}}{\mu_{h}+\gamma_{h}+\delta_{h}}+\frac{\beta_{2} \beta_{3} s_{h}^{*}\left(1-i_{v}^{*}\right)}{\mu_{v}\left(\mu_{h}+\gamma_{h}+\delta_{h}\right)}, & \text { if } R_{0}>1 .\end{cases}
\end{gathered}
$$

From (2) we have for $t \rightarrow \infty$

$$
\begin{aligned}
\frac{d N_{h}}{d t} & =N_{h}\left(b_{1}-\mu_{h}-\delta_{h} i_{h}\right) \\
& \longrightarrow \begin{cases}N_{h}\left(b_{1}-\mu_{h}\right), & \text { if } R_{0} \leq 1 \\
N_{h}\left(b_{1}-\mu_{h}-\delta_{h} i_{h}{ }^{*}\right), & \text { if } R_{0}>1 .\end{cases}
\end{aligned}
$$

By the definition of $R_{1}$, we have following threshold result.

Theorem A.1. The total population $N_{h}(t)$ for the system (1) decreases to zero if $R_{1}<1$ and increases to $\infty$ if $R_{1}>1$ as $t \rightarrow \infty$. The asymptotic rate of decrease is $\mu_{h}\left(R_{1}-1\right)$ if $R_{0} \leq 1$, and the asymptotic rate of increase is $\left(\mu_{h}+\delta_{h} i_{h}{ }^{*}\right)\left(R_{1}-1\right)$ when $R_{0}>1$.

Theorem A.2. Suppose $R_{1}>1$, for $t \rightarrow \infty,\left(S_{h}(t), I_{h}(t)\right.$, $\left.R_{h}(t)\right)$ tend to $(\infty, 0,0)$ if $R_{2}<1$ and tend to $(\infty, \infty, \infty)$ if $R_{2}>1$. 
TABLE 1: Asymptotic behavior with threshold criteria.

\begin{tabular}{lccccc}
\hline$R_{0}$ & $R_{1}$ & $R_{2}$ & $N_{h}$ & $\left(s_{h}, i_{h}, r_{h}, i_{v}\right) \rightarrow$ & $\left(S_{h}, I_{h}, R_{h}\right) \rightarrow$ \\
\hline$\leq 1$ & $=1, \delta_{h}=0$ & $\leq 1$ & $N_{h}=N_{h 0}$ & $(1,0,0,0)$ & $\left(N_{h 0}, 0,0\right)$ \\
$>1$ & $1, \delta_{h}=0$ & $=1$ & $N_{h}=N_{h 0}$ & $\left(s_{h}^{*}, i_{h}^{*}, r_{h}^{*}, i_{v}^{*}\right)$ & $(1,0,0,0)$ \\
$\leq 1$ & $<1$ & $<1$ & $N_{h} \rightarrow 0$ & $\left(s_{h}^{*}, i_{h}^{*}, r_{h}^{*}, i_{v}^{*}\right)$ & $(0,0,0)$ \\
$>1$ & $<1$ & $<1$ & $N_{h} \rightarrow 0$ & $(1,0,0,0)$ & $(0,0,0)$ \\
$\leq 1$ & $>1$ & $>1$ & $N_{h} \rightarrow \infty$ & $(1,0,0,0)$ & $(\infty, 0,0)$ \\
$\leq 1$ & $>1$ & $<1$ & $N_{h} \rightarrow \infty$ & $(1,0,0,0)$ & $\left(N_{h}^{*}, 0,0\right)$ \\
$<1$ & $=1$ & $>1$ & $N_{h} \rightarrow N_{h}^{*}$ & $\left(s_{h}^{*}, i_{h}^{*}, r_{h}^{*}, i_{v}^{*}\right)$ & $(\infty, \infty)$ \\
$>1$ & $>1$ & $N_{h} \rightarrow \infty$ & $\left(s_{h}^{*}, i_{h}^{*}, r_{h}^{*}, i_{v}^{*}\right)$ & $\left(s_{h}^{*}, i_{h}^{*}, r_{h}^{*}\right)$ \\
$>1$ & $=1$ & $N_{h} \rightarrow N_{h}^{*}$ & &
\end{tabular}

Proof. Since $i_{v}^{\prime} \rightarrow 0$ as $t \rightarrow \infty$, so in the limiting case the proportion of infectious mosquitoes is related to the proportion of infectious humans as

$$
i_{v}=\frac{\beta_{3}\left(1-i_{v}\right) i_{h}}{\mu_{v}}
$$

thus, the equation for $I_{h}(t)$ has limiting form

$$
\frac{d I_{h}(t)}{d t}=\left(\mu_{h}+\gamma_{h}+\delta_{h}\right)\left(R_{2}-1\right) I_{h},
$$

which shows that $I_{h}(t)$ decreases exponentially if $R_{2}<1$ and increases exponentially if $R_{2}>1$.

The solution $R_{h}(t)$ is given by

$$
R_{h}(t)=R_{h 0} e^{-\mu_{h} t}+\gamma_{h} e^{-\mu_{h} t} \int_{0}^{t} I_{h}(s) e^{\mu_{h} s} d s .
$$

From the exponential nature of $I_{h}(t)$, it follows that $I_{h}(t)$ declines exponentially if $R_{2}<1$ and grows exponentially if $R_{2}>1$.

Suppose $R_{1}=1$, then $b_{1}=\mu_{h}$ corresponding to $R_{0}<1$ and the differential equation for $N_{h}(t)$ will have the form

$$
\frac{d N_{h}}{d t}=-\delta_{h} I_{h}
$$

which means that $N_{h}(t)$ is bounded for all $t>0$, the equilibria $\left(N_{h}^{*}, 0,0,0\right)$ have one eigenvalue zero, and the other eigenvalues have negative real parts. Therefore, each orbit approaches an equilibrium point.

If $R_{0}>1$, the disease becomes "endemic." From the global stability of $E^{*}$ and the equation

$$
\frac{d N_{h}}{d t}=\delta_{h}\left[\left(\frac{b_{1}-\mu_{h}}{\delta_{h}}-i_{h}^{*}\right)-\left(i_{h}-i_{h}^{*}\right)\right] N_{h},
$$

we observe that $\left(N_{h}, S_{h}, I_{h}, R_{h}, I_{v}\right)$ approaches to $(0,0,0,0,0)$ or $(\infty, \infty, \infty, \infty, \infty)$ if $R_{1}<1$ or $R_{1}>1$. From the global stability of $i_{h}{ }^{*}$, we have $N_{h}(t)$ converges to some $N_{h}{ }^{*}$ as $t$ approaches to $\infty$. Since $s_{h}=S_{h} / N_{h}, i_{h}=I_{h} / N_{h}, r_{h}=R_{h} / N_{h}$, so we have $S_{h}{ }^{*}=s_{h}{ }^{*} N_{h}{ }^{*}, I_{h}{ }^{*}=i_{h}{ }^{*} N_{h}{ }^{*}, R_{h}{ }^{*}=r_{h}{ }^{*} N_{h}{ }^{*}$. All the above discussion is summarized in Table 1.

\section{Acknowledgment}

This work was supported by Basic Science Research Program through the National Research Foundation of Korea (NRF) funded by the Ministry of Education, Science and Technology (MEST) (2012-000599).

\section{References}

[1] H. Yang, H. Wei, and X. Li, "Global stability of an epidemic model for vector-borne disease," Journal of Systems Science \& Complexity, vol. 23, no. 2, pp. 279-292, 2010.

[2] Z. Feng and J. X. Velasco-Hernández, "Competitive exclusion in a vector-host model for the dengue fever," Journal of Mathematical Biology, vol. 35, no. 5, pp. 523-544, 1997.

[3] Z. Qiu, "Dynamical behavior of a vector-host epidemic model with demographic structure," Computers \& Mathematics with Applications, vol. 56, no. 12, pp. 3118-3129, 2008.

[4] V. Wiwanitkit, "Unusual mode of transmission of dengue," The Journal of Infection in Developing Countries, vol. 30, pp. 51-54, 2009.

[5] H.-M. Wei, X.-Z. Li, and M. Martcheva, "An epidemic model of a vector-borne disease with direct transmission and time delay," Journal of Mathematical Analysis and Applications, vol. 342, no. 2, pp. 895-908, 2008.

[6] L. Cai and X. Li, "Analysis of a simple vector-host epidemic model with direct transmission," Discrete Dynamics in Nature and Society, vol. 2010, Article ID 679613, 12 pages, 2010.

[7] A. A. Lashari and G. Zaman, "Global dynamics of vector-borne diseases with horizontal transmission in host population," Computers \& Mathematics with Applications, vol. 61, no. 4, pp. 745-754, 2011.

[8] M. de la Sen, R. P. Agarwal, A. Ibeas, and S. Alonso-Quesada, "On the existence of equilibrium points, boundedness, oscillating behavior and positivity of a SVEIRS epidemic model under constant and impulsive vaccination," Advances in Difference Equations, vol. 2011, Article ID 748608, 32 pages, 2011.

[9] M. de la Sen and S. Alonso-Quesada, "Vaccination strategies based on feedback control techniques for a general SEIRepidemic model," Applied Mathematics and Computation, vol. 218, no. 7, pp. 3888-3904, 2011.

[10] T. Zhang, J. Liu, and Z. Teng, "Dynamic behavior for a nonautonomous SIRS epidemic model with distributed delays," Applied Mathematics and Computation, vol. 214, no. 2, pp. 624631, 2009. 
[11] J. L. Aron and R. M. May, The Population Dynamics of Infectious Diseases, Chapman \& Hall, London, UK, 1982.

[12] H. W. Hethcote, "The mathematics of infectious diseases," SIAM Review, vol. 42, no. 4, pp. 599-653, 2000.

[13] G. A. Ngwa and W. S. Shu, "A mathematical model for endemic malaria with variable human and mosquito populations," Mathematical and Computer Modelling, vol. 32, no. 7-8, pp. 747-763, 2000.

[14] L. Esteva and C. Vargas, "A model for dengue disease with variable human population," Journal of Mathematical Biology, vol. 38, no. 3, pp. 220-240, 1999.

[15] M. Ozair, A. A. Lashari, I. H. Jung, and K. O. Okosun, "Stability analysis and optimal control of a vector-borne disease with nonlinear incidence," Discrete Dynamics in Nature and Society, vol. 2012, 21 pages, 2012.

[16] J. P. LaSalle, The Stability of Dynamical Systems, Regional Conference Series in Applied Mathematics, SIAM, Philadelphia, Pa, USA, 1976.

[17] M. Y. Li and J. S. Muldowney, "A geometric approach to globalstability problems," SIAM Journal on Mathematical Analysis, vol. 27, no. 4, pp. 1070-1083, 1996.

[18] X.-Q. Zhao, Dynamical Systems in Population Biology, vol. 16 of CMS Books in Mathematics, Springer, New York, NY, USA, 2003.

[19] G. Butler, H. I. Freedman, and P. Waltman, "Uniformly persistent systems," Proceedings of the American Mathematical Society, vol. 96, no. 3, pp. 425-430, 1986. 


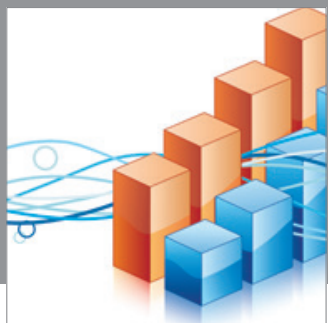

Advances in

Operations Research

mansans

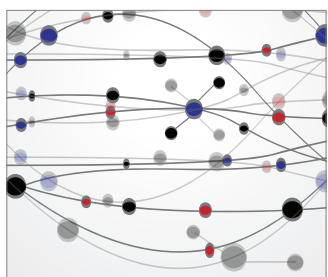

The Scientific World Journal
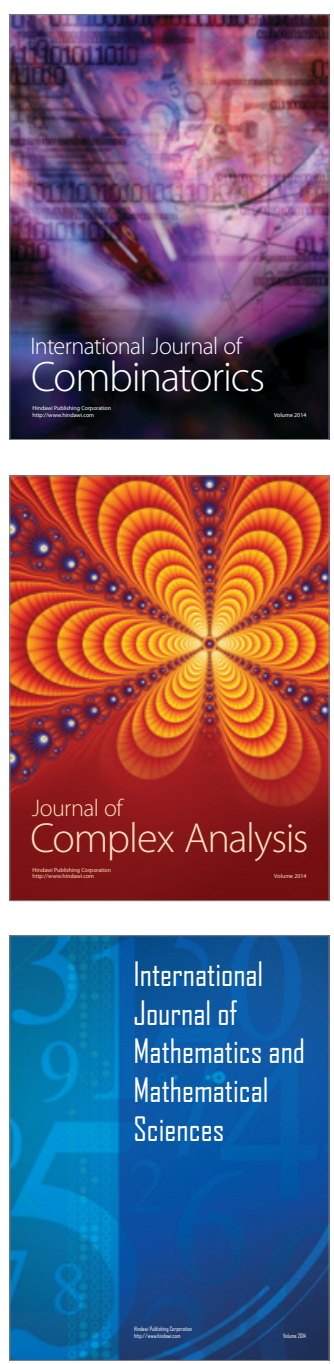
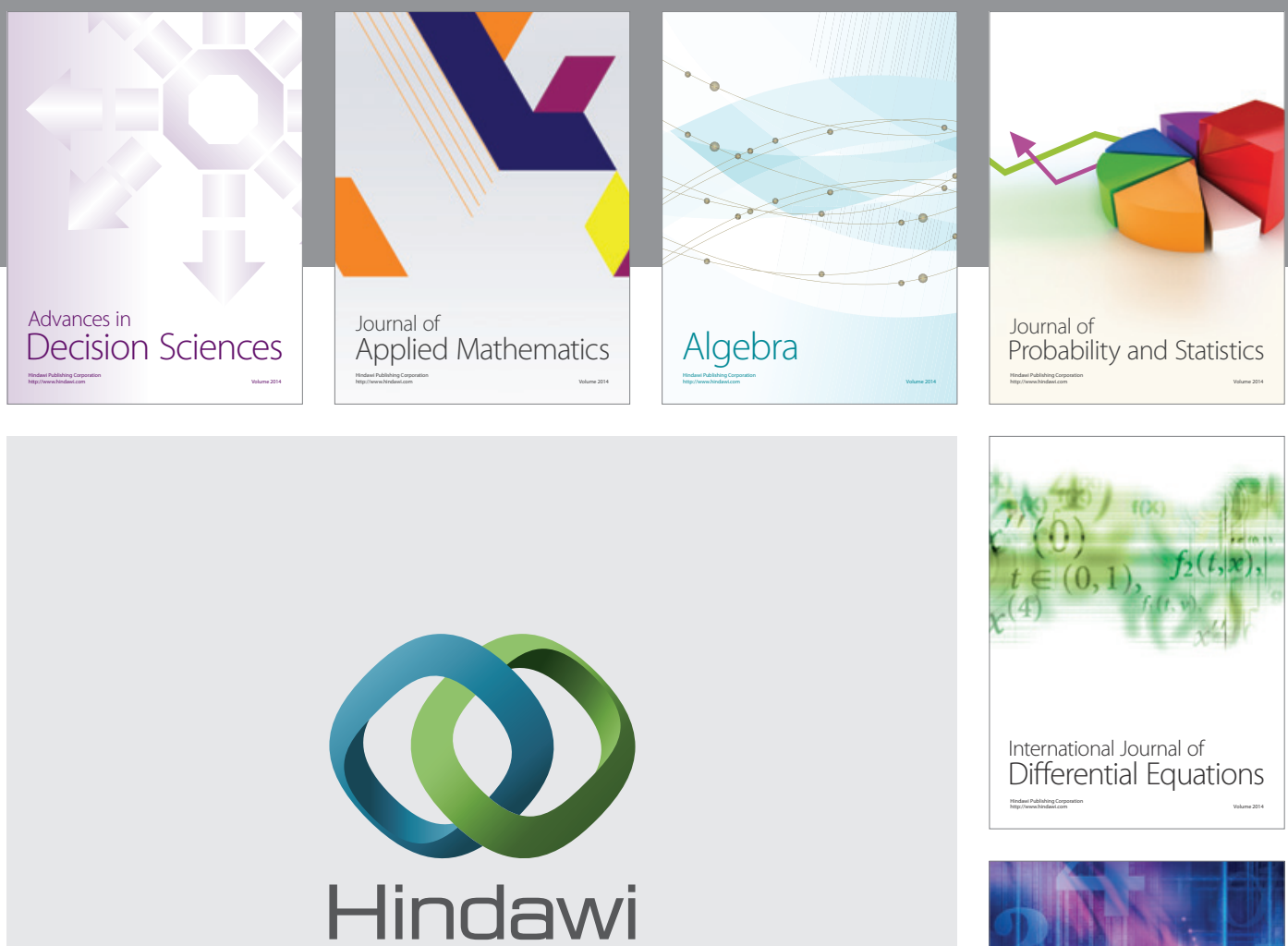

Submit your manuscripts at http://www.hindawi.com
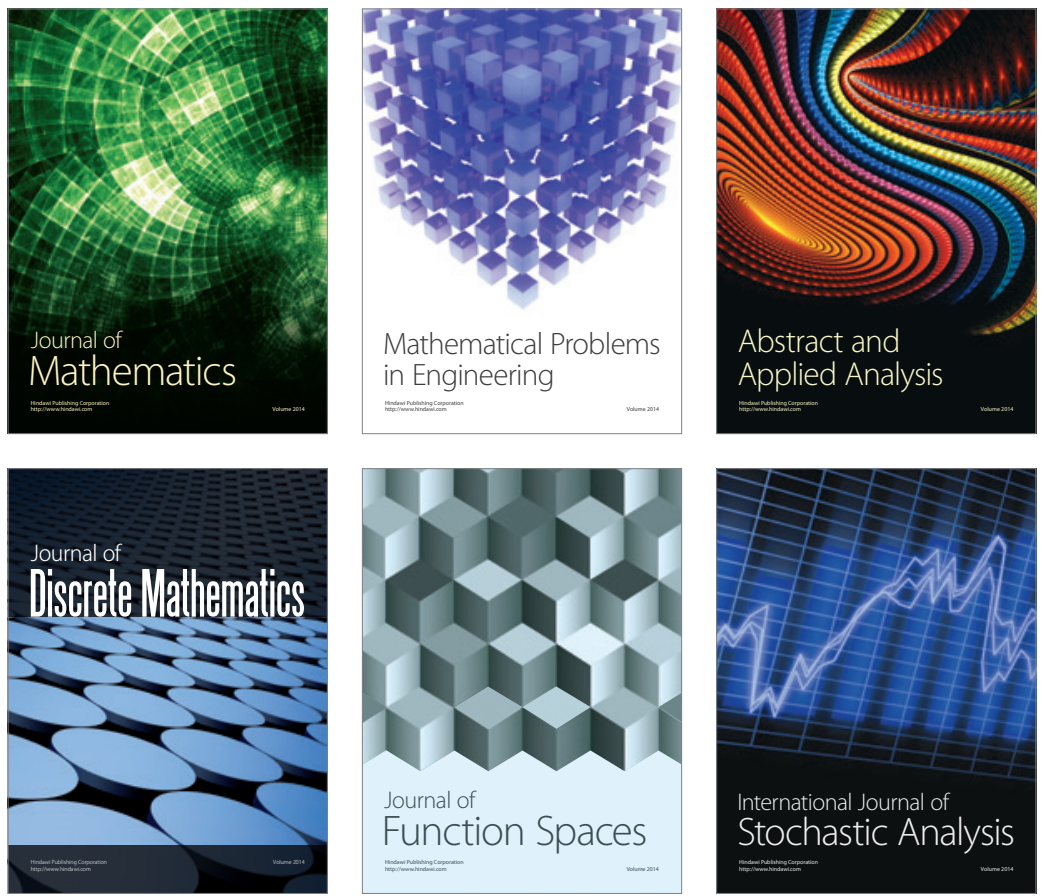

Journal of

Function Spaces

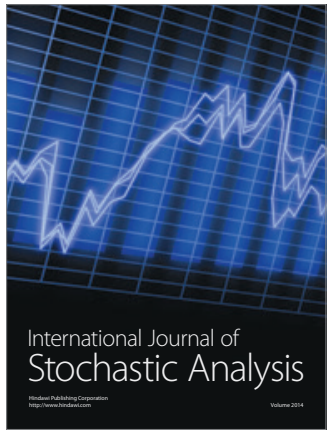

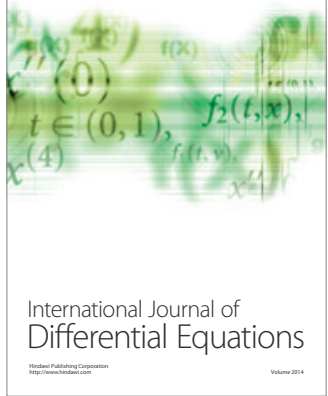
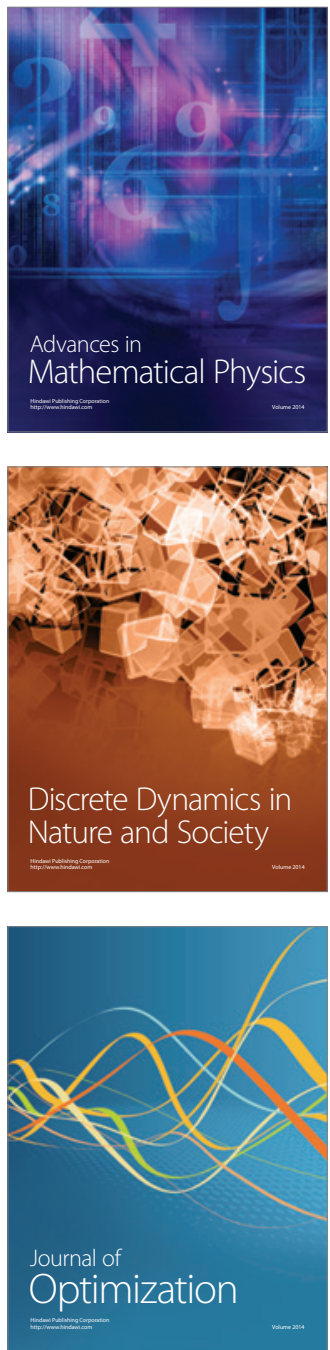